TRANSACTIONS OF THE

AMERICAN MATHEMATICAL SOCIETY

Volume 359, Number 2, February 2007, Pages 741-765

S 0002-9947(06)03909-2

Article electronically published on August 24, 2006

\title{
TILTING OBJECTS IN ABELIAN CATEGORIES AND QUASITILTED RINGS
}

\author{
RICCARDO COLPI AND KENT R. FULLER
}

\begin{abstract}
D. Happel, I. Reiten and S. Smalø initiated an investigation of quasitilted artin $K$-algebras that are the endomorphism rings of tilting objects in hereditary abelian categories whose Hom and Ext groups are all finitely generated over a commutative artinian ring $K$. Here, employing a notion of *-objects, tilting objects in arbitrary abelian categories are defined and are shown to yield a version of the classical tilting theorem between the category and the category of modules over their endomorphism rings. This leads to a module theoretic notion of quasitilted rings and their characterization as endomorphism rings of tilting objects in hereditary cocomplete abelian categories.
\end{abstract}

Tilting modules for finite-dimensional and artin algebras $A$ and the resulting tilting theorem between mod- $A$ and the finitely generated modules over the endomorphism ring of a tilting $A$-module were introduced by Brenner and Butler [3] and Happel and Ringel [15] as a generalization of the Morita equivalence theorem between categories of modules over a pair of algebras. A particularly tractable account was given by Bongartz in 2. Subsequently, Miyashita 19] and Colby and Fuller 4 showed that if $A$ is an arbitrary ring and $V_{A}$ is a tilting module, then the tilting theorem holds between $\operatorname{Mod}-A$ and $\operatorname{Mod}-R$, where $R=\operatorname{End}\left(V_{A}\right)$. The tilting theorem is basically a pair of equivalences $\mathcal{T} \rightleftarrows \mathcal{Y}$ and $\mathcal{F} \rightleftarrows \mathcal{X}$ between the members of torsion pairs $(\mathcal{T}, \mathcal{F})$ of $A$-modules and $(\mathcal{X}, \mathcal{Y})$ of $R$-modules. Particularly useful, from a representation theory point of view, is the case in which $A$ is hereditary, for then $(\mathcal{X}, \mathcal{Y})$ splits. In this case $R$ is said to be tilted.

Given a commutative artinian ring $K$, a locally finite abelian $K$-category $\mathcal{A}$ is an abelian category in which the Hom and Ext groups are $K$-modules of finite length and composition of morphisms is $K$-bilinear. Happel, Reiten and Smalø [14] defined a quasitilted (artin) algebra as the endomorphism algebra of a tilting object in a hereditary locally finite abelian $K$-category. They characterized quasitilted algebras as those with a split torsion pair $(\mathcal{X}, \mathcal{Y})$ in $\bmod -R$ such that $R_{R} \in \mathcal{Y}$ and $\operatorname{proj} \operatorname{dim} \mathcal{Y} \leq 1$, and showed that then $\operatorname{inj} \operatorname{dim} \mathcal{X} \leq 1$ and $\operatorname{gl} \operatorname{dim} R \leq 2$. They also characterized these algebras as those with global dimension $\leq 2$ such that every finitely generated indecomposable module has either injective or projective dimension $\leq 1$.

Here, following [13, we say that $R$ is a (right) quasitilted ring if it has split torsion pair $(\mathcal{X}, \mathcal{Y})$ in $\operatorname{Mod}-R$ such that $R_{R} \in \mathcal{Y}$ and $\operatorname{proj} \operatorname{dim} \mathcal{Y} \leq 1$. As we shall show, quasitilted rings turn out to be precisely the endomorphism rings of tilting objects

Received by the editors September 21, 2004 and, in revised form, December 3, 2004.

2000 Mathematics Subject Classification. Primary 16E10, 16G99, 16S50, 18E40, 18E25, 18G20; Secondary 16B50, 16D90.

(C)2006 American Mathematical Society Reverts to public domain 28 years from publication 
in hereditary cocomplete (i.e., with arbitrary coproducts) abelian categories, and they satisfy $\operatorname{inj} \operatorname{dim} \mathcal{X} \leq 1$ and $\operatorname{rtgl} \operatorname{dim} R \leq 2$.

When they introduced quasitilted algebras, Happel, Reiten and Smalø [14] showed that a tilting object $V$ in an abelian $K$-category $\mathcal{A}$ induces a pair of equivalences between torsion theories in $\mathcal{A}$ and mod- $R$ for the artin algebra $R=\operatorname{End}_{\mathcal{A}}(V)$; and, conversely, they proved that if $R$ is an artin algebra and $(\mathcal{X}, \mathcal{Y})$ is a torsion theory in $\bmod -R$ such that $R_{R} \in \mathcal{Y}$, then there is a locally finite abelian $K$-category $\mathcal{A}$ with a tilting object $V$ such that $R=\operatorname{End}_{\mathcal{A}}(V)$ and $(\mathcal{X}, \mathcal{Y})$ is given by $V$.

In [7] Colpi, employing the notion of a $*$-object (a version of the $*$-modules of Menini and Orsatti [17]), proved that a tilting object $V$ in a Grothendieck category $\mathcal{G}$ induces a tilting theorem between $\mathcal{G}$ and $\operatorname{Mod}-R$, for $R=\operatorname{End}_{\mathcal{G}}(V)$. In order to prove our characterization of quasitilted rings, we employ a similar approach to show that a tilting object in a cocomplete abelian category $\mathcal{A}$ induces a tilting theorem between $\mathcal{A}$ and the category of right modules over its endomorphism ring; and using an argument similar to one in [14, we show conversely that if $(\mathcal{X}, \mathcal{Y})$ is a torsion theory in Mod- $R$ with $R_{R} \in \mathcal{Y}$, then there is a tilting object $V$ in a cocomplete abelian category $\mathcal{A}$ such that $R=\operatorname{End}_{\mathcal{A}}(V)$ and $(\mathcal{X}, \mathcal{Y})$ is given by $V$.

Our concluding sections contain an example of a non-noetherian quasitilted ring that is not tilted, some open questions, and an appendix providing needed results on the behavior of coproducts under the functor $\operatorname{Ext}_{\mathcal{A}}^{1}(-, L)$ for an abelian category $\mathcal{A}$.

\section{Maximal equivalences}

In the sequel, $\mathcal{A}$ denotes a fixed abelian category and $V$ an object of $\mathcal{A}$ such that $V^{(\alpha)}$ exists in $\mathcal{A}$ for any cardinal $\alpha$.

Proposition 1.1. Let $R=\operatorname{End}_{\mathcal{A}}(V), H_{V}=\operatorname{Hom}_{\mathcal{A}}(V,-): \mathcal{A} \rightarrow \operatorname{Mod}-R$. Then $H_{V}$ has a left adjoint additive functor $T_{V}: \operatorname{Mod}-R \rightarrow \mathcal{A}$ such that $T_{V}(R)=V$. Let $\sigma: 1_{\mathrm{Mod}-R} \rightarrow H_{V} T_{V}$ and $\rho: T_{V} H_{V} \rightarrow 1_{\mathcal{A}}$ be respectively the unit and the counit of the adjunction $\left\langle T_{V}, H_{V}\right\rangle$. Let us define

$$
\begin{gathered}
\operatorname{Tr}_{V}: \mathcal{A} \rightarrow \mathcal{A} \text { by } \operatorname{Tr}_{V}(M)=\sum\left\{\operatorname{Im} f \mid f \in \operatorname{Hom}_{\mathcal{A}}(V, M)\right\}, \\
\operatorname{Ann}_{V}: \operatorname{Mod}-R \rightarrow \text { Mod- } R \text { by } \operatorname{Ann}_{V}(N)=\sum\left\{L \mid L \stackrel{i}{\hookrightarrow} N, T_{V}(i)=0\right\}
\end{gathered}
$$

and

$$
\text { Gen } V=\left\{M \in \mathcal{A} \mid \operatorname{Tr}_{V}(M)=M\right\}, \quad \text { Faith } V=\left\{N \in \operatorname{Mod}-R \mid \operatorname{Ann}_{V}(N)=0\right\} .
$$

Then:

a) The canonical inclusion $\operatorname{Tr}_{V}(M) \hookrightarrow M$ induces a natural isomorphism $H_{V}\left(\operatorname{Tr}_{V}(M)\right) \cong M$, and the canonical projection $N \rightarrow N / \operatorname{Ann}_{V}(N)$ induces a natural isomorphism $T_{V}(N) \cong T_{V}\left(N / \operatorname{Ann}_{V}(N)\right)$.

b) $\operatorname{Tr}_{V}$ is an idempotent preradical, and $\mathrm{Ann}_{V}$ is a radical.

c) $\operatorname{Tr}_{V}(M)=\operatorname{Im} \rho_{M}$, and $\operatorname{Ann}_{V}(N)=\operatorname{Ker} \sigma_{N}$.

d) $T_{V}(\operatorname{Mod}-R) \subseteq$ Gen $V$, and $H_{V}(\mathcal{A}) \subseteq$ Faith $V$.

e) Gen $V$ is closed under (existing) coproducts and factors in $\mathcal{A}$, and Faith $V$ is closed under products and submodules in Mod- $R$.

Proof. The first part of the statement was proved by Popescu in [20, Corollary 7.3 and Note 1 on page 109. 
a) The first part is clear, since $\operatorname{Im}(f) \subseteq \operatorname{Tr}_{V}(M)$ for any $f \in \operatorname{Hom}_{\mathcal{A}}(V, M)$. Now let $\operatorname{Ann}_{V}(N)=\sum\left\{L_{\lambda} \mid \lambda \in \Lambda\right\}$, with $j_{\lambda}: L_{\lambda} \hookrightarrow \operatorname{Ann}_{V}(N)$, for each $i_{\lambda}: L_{\lambda} \hookrightarrow N$ with $T_{V}\left(i_{\lambda}\right)=0$. Applying the functor $T_{V}$ to the commutative diagram

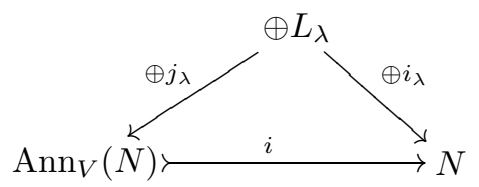

we immediately obtain $T_{V}(i)=0$, since $\oplus j_{\lambda}$ is an epimorphism and $T_{V}$ is right exact and commutes with direct sums. Therefore, if we apply $T_{V}$ to the exact sequence

$$
0 \rightarrow \operatorname{Ann}_{V}(N) \stackrel{i}{\rightarrow} N \stackrel{\pi}{\rightarrow} N / \operatorname{Ann}_{V}(N) \rightarrow 0
$$

we obtain the exact sequence

$$
T_{V}\left(\operatorname{Ann}_{V}(N)\right) \stackrel{0}{\rightarrow} T_{V}(N) \stackrel{T_{V}(\pi)}{\longrightarrow} T_{V}\left(N / \operatorname{Ann}_{V}(N)\right) \rightarrow 0
$$

which shows that $T_{V}(\pi)$ is an isomorphism.

b) The first part is clear. Moreover, using the right exactness of $T_{V}$ and, for $i_{\lambda}: L_{\lambda} \hookrightarrow M$ and $f: M \rightarrow N$, using the commutative diagram

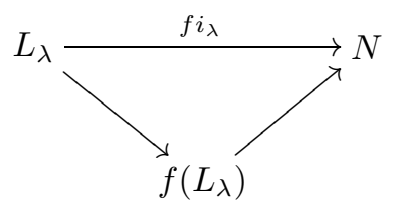

it becomes clear that $\mathrm{Ann}_{V}$ is a preradical. Let us prove that $\mathrm{Ann}_{V}$ is a radical, i.e., $\operatorname{Ann}_{V}\left(N / \operatorname{Ann}_{V}(N)\right)=0$. Let $\operatorname{Ann}_{V}(N) \leq L \leq N$ such that $T_{V}(i)=0$ for $i: L / \operatorname{Ann}_{V}(N) \hookrightarrow N / \operatorname{Ann}_{V}(N)$. Applying $T_{V}$ to the commutative diagram

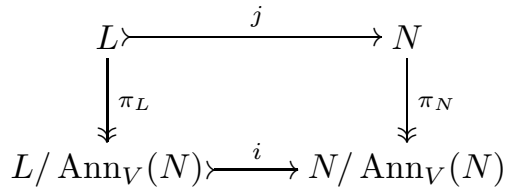

we have $0=T_{V}(i) T_{V}\left(\pi_{L}\right)=T_{V}\left(i \pi_{L}\right)=T_{V}\left(\pi_{N} j\right)=T_{V}\left(\pi_{N}\right) T_{V}(j)$, so that $T_{V}(j)=$ 0 , since $T_{V}\left(\pi_{N}\right)$ is an isomorphism by a). This gives $L \leq \operatorname{Ann}_{V}(N)$, as desired.

c) $\operatorname{Im} \rho_{M}$ is a factor of $T_{V} H_{V}(M)$, and $T_{V} H_{V}(M) \in T_{V}(\operatorname{Mod}-R)=T_{V}(\operatorname{Gen} R)$ $\subseteq$ Gen $T_{V}(R)=$ Gen $V$ since $T_{V}$ is right exact and preserves coproducts. Therefore $\operatorname{Im} \rho_{M} \in \operatorname{Gen} V$, i.e., $\operatorname{Im} \rho_{M} \subseteq \operatorname{Tr}_{V}(M)$. Conversely, let $V^{(\alpha)} \stackrel{\varphi}{\rightarrow} M$ be a morphism such that $\operatorname{Im} \varphi=\operatorname{Tr}_{V}(M)$. In the commutative diagram

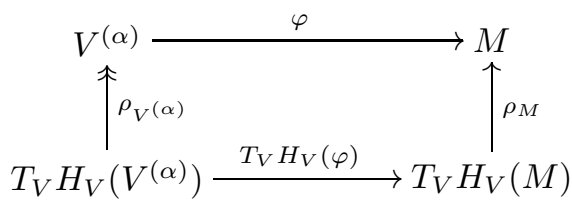

$\rho_{V^{(\alpha)}}$ is epi-split by adjointness, since $V^{(\alpha)}=T_{V}\left(R^{(\alpha)}\right)$. Thus $\operatorname{Tr}_{V}(M)=\operatorname{Im} \varphi \leq$ $\operatorname{Im} \rho_{M}$, and so they are equal. 
Now let $N \in \operatorname{Mod}-R$. From the commutative diagram

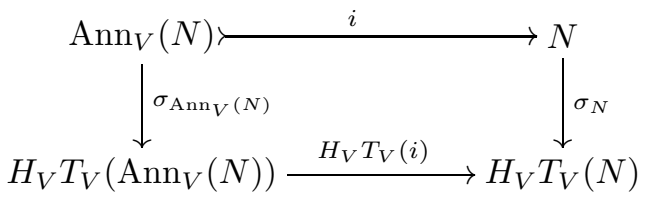

since $T_{V}(i)=0$ (as in the proof of a)), we see that $\sigma_{N} i=0$, i.e., $\operatorname{Ann}_{V}(N) \leq$ $\operatorname{Ker} \sigma_{N}$. Conversely, if $i: \operatorname{Ker} \sigma_{N} \hookrightarrow N$ is the canonical inclusion, then $\sigma_{N} i=0$, so that $T_{V}\left(\sigma_{N}\right) T_{V}(i)=0$, and so $T_{V}(i)=0$, since $T_{V}\left(\sigma_{N}\right)$ is mono-split by adjointness. This proves that $\operatorname{Ker} \sigma_{N} \leq \operatorname{Ann}_{V}(N)$, and so they are equal.

d) By c), it follows that $M \in$ Gen $V$ if and only if $\rho_{M}$ is epic, and $N \in$ Faith $V$ if and only if $\sigma_{N}$ is monic. Since by adjointness $\rho_{M}$ is epi-split for any $M \in$ $T_{V}(\operatorname{Mod}-R)$, and $\sigma_{N}$ is mono-split for any $N \in H_{V}(\mathcal{A})$, d) follows.

e) follows from b), thanks to [22, Ch. VI, Proposition 1.4.

Remark 1.2. From the statements a), b), c) and d) in Proposition 1.1 it follows that Gen $V \subseteq \mathcal{A}$ and Faith $V \subseteq \operatorname{Mod}-R$ are the largest full subcategories between which the adjunction $\left\langle T_{V}, H_{V}\right\rangle$ can induce an equivalence.

This suggests the following.

Definition 1.3. $V \in \mathcal{A}$ is called a $*$-object if $\left\langle T_{V}, H_{V}\right\rangle$ induces an equivalence

$$
H_{V}: \text { Gen } V \rightleftarrows \text { Faith } V: T_{V} \text {. }
$$

Note that Gen $V$ is closed under factors and coproducts, and Faith $V$ is closed under submodules and direct products, thanks to Proposition 1.1 k). These properties, together with the equivalence, characterize $*$-objects, in view of the following version of Menini and Orsatti's theorem [17] (see also [5], section 2).

Theorem 1.4. Let $\mathcal{A}$ be a cocomplete abelian category, and let $R$ be a ring. Let $\mathcal{G} \subseteq \mathcal{A}$ be a full subcategory closed under factors and coproducts, and let $\mathcal{F} \subseteq \operatorname{Mod}-R$ be a full subcategory closed under submodules and direct products, and suppose that there is a category equivalence

$$
H: \mathcal{G} \rightleftarrows \mathcal{F}: T \text {. }
$$

Let $\bar{R}=R / \mathbf{r}_{R}(\mathcal{F})$. Then $\bar{R}_{R}$ is in $\mathcal{F}$, and setting $V=T(\bar{R})$ we have natural isomorphisms $H \cong H_{V}$ and $T \cong T_{V}$, and equalities $\mathcal{G}=$ Gen $V$ and $\mathcal{F}=$ Faith $V$. In particular, $V$ is a $*$-object in $\mathcal{A}$ and $\bar{R} \cong \operatorname{End}_{\mathcal{A}}(V)$.

Proof. Since $\mathcal{F}$ is closed under submodules and products, $\bar{R}_{R}$ is in $\mathcal{F}$. For any $M \in \mathcal{G}$ we have $H(M) \cong \operatorname{Hom}_{R}(\bar{R}, H(M)) \cong \operatorname{Hom}_{\mathcal{A}}(V, M)$ canonically in Mod- $R$.

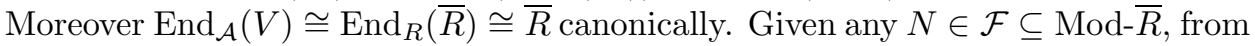
the exact sequence $\bar{R}^{(\alpha)} \rightarrow N \rightarrow 0$ we obtain the exact sequence $V^{(\alpha)} \rightarrow T_{V}(N) \rightarrow 0$ which gives $T_{V}(N) \in \mathcal{G}$, since $\mathcal{G}$ is closed under coproducts and factors. Therefore $T \cong T_{V}$, as both functors are left adjoint to $H \cong H_{V}$. From statement c) in Proposition 1.1 we derive the inclusions $\mathcal{G} \subseteq$ Gen $V$ and $\mathcal{F} \subseteq$ Faith $V$. On the other hand, $V \in \mathcal{G}$ and the closure properties of $\mathcal{G}$ immediately give Gen $V \subseteq \mathcal{G}$. Moreover, if $N \in$ Faith $V$, then from statements b) and c) in Proposition 1.1 we derive $N \stackrel{\sigma_{N}}{\hookrightarrow} H_{V} T_{V}(N) \in H_{V}(\operatorname{Gen} V)=H_{V}(\mathcal{G})=H(\mathcal{G}) \subseteq \mathcal{F}$, hence $N \in \mathcal{F}$ by the closure properties of $\mathcal{F}$. This shows that Faith $V \subseteq \mathcal{F}$. 
For convenience, we restate [7], Lemma 1.5.

Lemma 1.5. Let $\mathcal{A}$ and $\mathcal{B}$ be abelian categories, and let $\mathcal{G} \subseteq \mathcal{A}$ and $\mathcal{F} \subseteq \mathcal{B}$ be full subcategories, each one of which is either closed under subobjects or factor objects. Let $\langle T, H\rangle$ be an adjoint pair of additive functors $\mathcal{G} \underset{T}{\stackrel{H}{\rightleftarrows}} \mathcal{F}$, with unit $\sigma: 1 \rightarrow H T$ and counit $\rho: T H \rightarrow 1$. Then:

a) If $\rho_{M}$ is an isomorphism for all $M \in \mathcal{G}$, then $T$ preserves the exactness of short exact sequences with objects in $H(\mathcal{G})$.

b) If $\sigma_{N}$ is an isomorphism for all $N \in \mathcal{F}$, then $H$ preserves the exactness of short exact sequences with objects in $T(\mathcal{F})$.

\section{Tilting objects}

Let $\mathcal{A}$ be an abelian category. Following Dickson [9], a torsion theory in $\mathcal{A}$ is a pair of classes of objects $(\mathcal{T}, \mathcal{F})$ of $\mathcal{A}$ such that

(1) $\mathcal{T}=\left\{T \in \mathcal{A} \mid \operatorname{Hom}_{\mathcal{A}}(T, F)=0 \forall F \in \mathcal{F}\right\}$,

(2) $\mathcal{F}=\left\{F \in \mathcal{A} \mid \operatorname{Hom}_{\mathcal{A}}(T, F)=0 \forall T \in \mathcal{T}\right\}$,

(3) for each $X \in \mathcal{A}$ there is a short exact sequence $0 \rightarrow T \rightarrow X \rightarrow F \rightarrow 0$, with $T \in \mathcal{T}$ and $F \in \mathcal{F}$.

Now let $V$ be an object of $\mathcal{A}$ such that $V^{(\alpha)} \in \mathcal{A}$ for any cardinal $\alpha$. We shall denote by Gen $V$ the full subcategory of $\mathcal{A}$ generated by $V$ and by $\overline{\text { Gen }} V$ the closure of Gen $V$ under subobjects: $\overline{\text { Gen }} V$ is the smallest exact abelian subcategory of $\mathcal{A}$ containing Gen $V$. Moreover we let Pres $V$ denote the full subcategory of Gen $V$ which consists of the objects in $\mathcal{A}$ presented by $V$, i.e., Pres $V=\{M \in \mathcal{A} \mid$ $\exists$ an exact sequence $\left.V^{(\beta)} \rightarrow V^{(\alpha)} \rightarrow M \rightarrow 0\right\}$. Finally, let $R=\operatorname{End}_{\mathcal{A}}(V)$ and

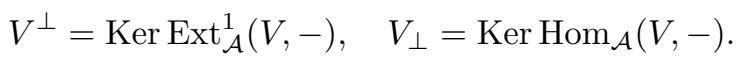

In this setting we have analogues of results regarding non-finitely generated tilting modules from [8] (see also [6], section 3.1).

Proposition 2.1. Let $V \in \mathcal{A}$.

a) If Gen $V \subseteq V^{\perp}$, then $\operatorname{Tr}_{V}$ is a radical. In particular (Gen $V, V_{\perp}$ ) is a torsion theory in $\mathcal{A}$.

b) If Gen $V=V^{\perp}$, then Gen $V=\operatorname{Pres} V$.

c) If $\overline{\operatorname{Gen}} V=\mathcal{A}$, then the equality Gen $V=V^{\perp}$ is equivalent to the following conditions:

i) $\operatorname{proj} \operatorname{dim} V \leq 1$,

ii) $\operatorname{Ext}_{\mathcal{A}}^{1}\left(V, V^{(\alpha)}\right)=0$ for any cardinal $\alpha$,

iii) if $M \in \mathcal{A}$ and $\operatorname{Hom}_{\mathcal{A}}(V, M)=0=\operatorname{Ext}_{\mathcal{A}}^{1}(V, M)$, then $M=0$.

Proof. a) Let $M \in \mathcal{A}$ and consider the canonical exact sequence

$$
0 \rightarrow \operatorname{Tr}_{V}(M) \rightarrow M \rightarrow M / \operatorname{Tr}_{V}(M) \rightarrow 0 .
$$

We obtain the exact sequence

$$
0 \rightarrow H_{V}\left(\operatorname{Tr}_{V}(M)\right) \stackrel{\cong}{\rightrightarrows} H_{V}(M) \rightarrow H_{V}\left(M / \operatorname{Tr}_{V}(M)\right) \rightarrow \operatorname{Ext}_{\mathcal{A}}^{1}\left(V, \operatorname{Tr}_{V}(M)\right)=0
$$

which shows that $H_{V}\left(M / \operatorname{Tr}_{V}(M)\right)=0$, i.e., $\operatorname{Tr}_{V}\left(M / \operatorname{Tr}_{V}(M)\right)=0$. This and Proposition 1.1b) prove that $\operatorname{Tr}_{V}$ is an idempotent radical. This shows that for any $M \in \mathcal{A}, \operatorname{Tr}_{V}(M)$ is the unique subobject of $M$ such that $\operatorname{Tr}_{V}(M) \in \operatorname{Gen} V$ and $M / \operatorname{Tr}_{V}(M) \in V_{\perp}$, and so $\left(\operatorname{Gen} V, V_{\perp}\right)$ is a torsion theory in $\mathcal{A}$. 
b) Let $M \in \operatorname{Gen} V$ and $\alpha=\operatorname{Hom}_{\mathcal{A}}(V, M)$. Then we have the exact sequences

$$
0 \rightarrow K \rightarrow V^{(\alpha)} \stackrel{\varphi}{\rightarrow} M \rightarrow 0
$$

and

$$
H_{V}\left(V^{(\alpha)}\right) \stackrel{H_{V}(\varphi)}{\longrightarrow} H_{V}(M) \rightarrow \operatorname{Ext}_{\mathcal{A}}^{1}(V, K) \rightarrow 0
$$

where the morphism $H_{V}(\varphi)$ is an epimorphism by construction. Therefore $\operatorname{Ext}_{\mathcal{A}}^{1}(V, K)=0$, so by assumption $K \in$ Gen $V$. This proves that $M \in \operatorname{Pres} V$.

c) Let $\overline{\operatorname{Gen}} V=\mathcal{A}$ and Gen $V=V^{\perp}$. Let us prove i), showing that $\operatorname{Ext}_{\mathcal{A}}^{2}(V, M)$ $=0$ for any $M \in \mathcal{A}$. Indeed, given a representative of an element $\epsilon \in \operatorname{Ext}_{\mathcal{A}}^{2}(V, M)$, say

$$
0 \rightarrow M \rightarrow E_{1} \stackrel{f}{\rightarrow} E_{2} \rightarrow V \rightarrow 0,
$$

let $I=\operatorname{Im} f$. Embedding $E_{1}$ in a suitable object $X \in$ Gen $V$, we first have a push-out diagram (dual to 22, Proposition 5.1, page 90)

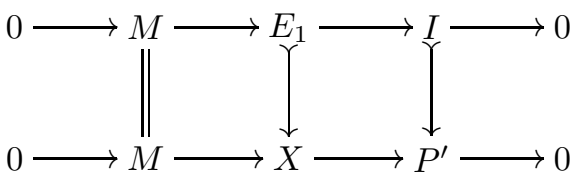

where $X$, and so $P^{\prime}$, are in Gen $V$. Then we have a second push-out diagram

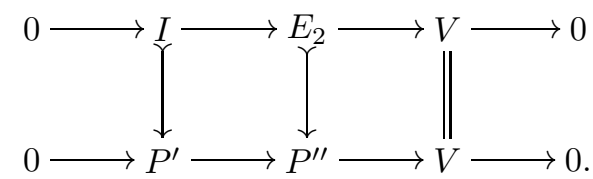

By glueing (1) and (2) together, we derive a commutative diagram with exact rows

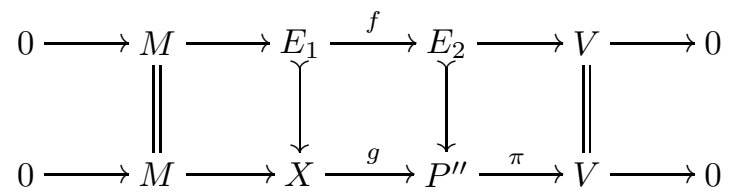

where $\operatorname{Im} g=P^{\prime} \in V^{\perp}$. Then $\pi$ is epi-split, and so $\epsilon \sim 0$. This proves i). Condition ii) is contained in the hypothesis, and condition iii) follows from a).

Conversely, let us assume that conditions i), ii) and iii) hold. The first condition assures that $V^{\perp}$ is closed under factors. Therefore, using the second condition we immediately see that Gen $V \subseteq V^{\perp}$. In order to prove the opposite inclusion, given any $M \in V^{\perp}$, from the exact sequence $0 \rightarrow \operatorname{Tr}_{V}(M) \rightarrow M \rightarrow M / \operatorname{Tr}_{V}(M) \rightarrow 0$ and using condition i) we obtain the exact sequence

$$
\begin{aligned}
0 \rightarrow \operatorname{Hom}_{\mathcal{A}}\left(V, \operatorname{Tr}_{V}(M)\right) \stackrel{\cong}{\cong} \operatorname{Hom}_{\mathcal{A}}(V, M) \rightarrow \operatorname{Hom}_{\mathcal{A}}\left(V, M / \operatorname{Tr}_{V}(M)\right) \\
\quad \rightarrow \operatorname{Ext}_{\mathcal{A}}^{1}\left(V, \operatorname{Tr}_{V}(M)\right)=0=\operatorname{Ext}_{\mathcal{A}}^{1}(V, M) \rightarrow \operatorname{Ext}_{\mathcal{A}}^{1}\left(V, M / \operatorname{Tr}_{V}(M)\right) \rightarrow 0 .
\end{aligned}
$$

Hence $\operatorname{Hom}_{\mathcal{A}}\left(V, M / \operatorname{Tr}_{V}(M)\right)=0=\operatorname{Ext}_{\mathcal{A}}^{1}\left(V, M / \operatorname{Tr}_{V}(M)\right)$. Now condition iii) gives $M / \operatorname{Tr}_{V}(M)=0$, i.e., $M=\operatorname{Tr}_{V}(M) \in \operatorname{Gen} V$. This proves that $V^{\perp} \subseteq$ Gen $V$.

Remark 2.2. If $\mathcal{A}$ is cocomplete with exact coproducts, or $\mathcal{A}$ has enough injectives, then $\overline{\mathrm{Gen}} V=\mathcal{A}$ whenever Gen $V=V^{\perp}$. 
Proof. If $\mathcal{A}$ has enough injectives, then every object of $\mathcal{A}$ embeds in an injective object which, by definition, belongs to $V^{\perp}=\operatorname{Gen} V$. Let us assume, now, that $\mathcal{A}$ is cocomplete with exact coproducts. Let $M \in \mathcal{A}$ and $\alpha$ be the cardinality of a spanning set for $\operatorname{Ext}_{\mathcal{A}}^{1}(V, M)$ as a right $R$-module. Then, arguing as in [6], Lemma 3.4.4, we can find an exact sequence

$$
0 \rightarrow M \rightarrow X \rightarrow V^{(\alpha)} \rightarrow 0
$$

such that the connecting homomorphism $\operatorname{Hom}_{\mathcal{A}}\left(V, V^{(\alpha)}\right) \rightarrow \operatorname{Ext}_{\mathcal{A}}^{1}(V, M)$ is onto. This gives $\operatorname{Ext}_{\mathcal{A}}^{1}(V, X)=0$, i.e., $X \in V^{\perp}=$ Gen $V$, and so it proves that $\overline{\mathrm{Gen}} V=$ $\mathcal{A}$.

In view of this last remark, we add a third condition to the Definition 2.3 of [7] to obtain

Definition 2.3. An object $V$ in an abelian category $\mathcal{A}$ that contains arbitrary coproducts of copies of $V$ is called a tilting object if:

i) $V$ is selfsmall (i.e., $\operatorname{Hom}_{\mathcal{A}}\left(V, V^{(\alpha)}\right) \cong R^{(\alpha)}$ for any cardinal $\alpha$ );

ii) Gen $V=V^{\perp}$

iii) $\overline{\mathrm{Gen}} V=\mathcal{A}$.

So, to any tilting object $V \in \mathcal{A}$ is naturally associated a torsion theory $(\mathcal{T}, \mathcal{F})$ in $\mathcal{A}$, namely $\mathcal{T}=V^{\perp}$ and $\mathcal{F}=V_{\perp}$.

Now we can extend [7, Theorem 3.2, as follows.

Theorem 2.4. Let $\mathcal{A}$ be an abelian category such that $V^{(\alpha)} \in \mathcal{A}$ for any cardinal $\alpha$. Then the following are equivalent:

(a) $V$ is a $*$-object;

(b) $V$ is a tilting object in $\overline{\mathrm{Gen}} V$;

(c) $\rho$ is monic in $\mathcal{A}$ and $\sigma$ is epic in $\operatorname{Mod}-R$;

(d) $V$ is selfsmall, Gen $V=\operatorname{Pres} V$ and $H_{V}$ preserves short exact sequences in $\mathcal{A}$ with all terms in Gen $V$;

(e) $V$ is selfsmall, and for any short exact sequence $0 \rightarrow L \rightarrow M \rightarrow N \rightarrow 0$ in $\mathcal{A}$ with $M$ (and $N$ ) in Gen $V$, the sequence $0 \rightarrow H_{V}(L) \rightarrow H_{V}(M) \rightarrow$ $H_{V}(N) \rightarrow 0$ is exact if and only if $L \in$ Gen $V$.

Proof. (a) $\Rightarrow$ (b) We see that $V$ is selfsmall, since $H_{V}\left(V^{(\alpha)}\right)=H_{V} T_{V}\left(R^{(\alpha)}\right) \cong$ $R^{(\alpha)}=H_{V}(V)^{(\alpha)}$ canonically. We can assume that $\mathcal{A}=\overline{\mathrm{Gen}} V$. In order to prove that Gen $V \subseteq V^{\perp}$, given any $M \in$ Gen $V$ we show that any short exact sequence in $\mathcal{A}$,

$$
0 \rightarrow M \rightarrow X \stackrel{\pi}{\rightarrow} V \rightarrow 0
$$

splits. Let $X \stackrel{i}{\hookrightarrow} L$ be a fixed embedding with $L \in$ Gen $V$, and let us consider the push-out diagram

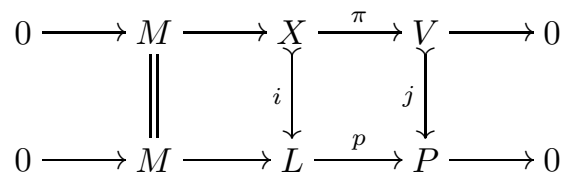


where the second row is in Gen $V$. From (2) we obtain the commutative diagram with exact rows

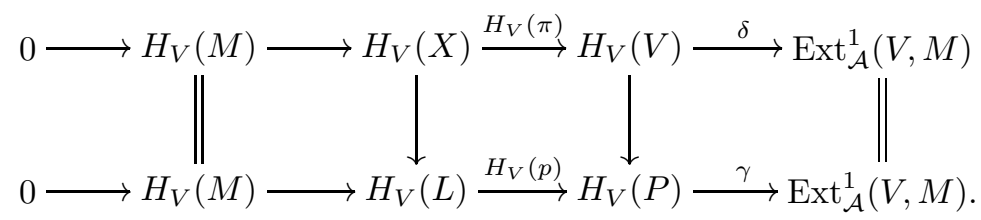

Since, from statement b) in Lemma 1.5 the morphism $H_{V}(p)$ in (3) is epic, we see that $\gamma=0$, so that $\delta=0$, too. This shows that $H_{V}(\pi)$ is epic, so that (1) splits.

Conversely, let us prove that $V^{\perp} \subseteq$ Gen $V$. Given any $M \in V^{\perp}$, let

$$
0 \rightarrow M \rightarrow X_{0} \stackrel{\varphi}{\rightarrow} X_{1} \rightarrow 0
$$

be a fixed exact sequence with $X_{0}\left(\right.$ and $\left.X_{1}\right)$ in Gen $V$. Since $\operatorname{Ext}_{\mathcal{A}}^{1}(V, M)=0$ by assumption, $H_{V}(\varphi)$ is epic. Therefore we have the commutative diagram with exact rows

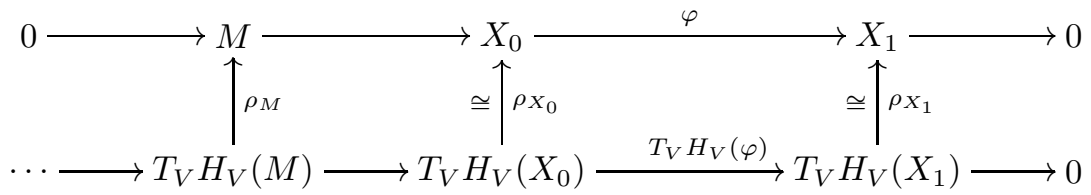

which shows that $\rho_{M}$ is epic, i.e., $M \in$ Gen $V$.

(b) $\Rightarrow$ (e) Assume that $0 \rightarrow L \rightarrow M \rightarrow N \rightarrow 0$ is an exact sequence in $\mathcal{A}$ with $M($ and $N)$ in Gen $V$. Then, since by assumption Gen $V=V^{\perp}$, the sequence $0 \rightarrow$ $H_{V}(L) \rightarrow H_{V}(M) \rightarrow H_{V}(N) \rightarrow \operatorname{Ext}_{\mathcal{A}}^{1}(V, L) \rightarrow 0$ is exact, and so $\operatorname{Ext}_{\mathcal{A}}^{1}(V, L)=0$ if and only if $L \in$ Gen $V$.

(e) $\Rightarrow$ (d) Let $M \in$ Gen $V$, and let $\alpha=H_{V}(M)$. Then there is a short exact sequence $0 \rightarrow K \rightarrow V^{(\alpha)} \stackrel{\varphi}{\rightarrow} M \rightarrow 0$ such that $H_{V}(\varphi)$ is epic. By hypothesis, we must have $K \in$ Gen $V$. This shows that $M \in \operatorname{Pres} V$.

(d) $\Rightarrow$ (c) Let $N \in \operatorname{Mod}-R$ and let $R^{(\beta)} \rightarrow R^{(\alpha)} \stackrel{\varphi}{\rightarrow} N \rightarrow 0$ be exact. Since $H_{V}$ is exact on Gen $V$ by assumption, it preserves the exactness of the sequence $0 \rightarrow K \rightarrow T_{V}\left(R^{(\alpha)}\right) \stackrel{T_{V}(\varphi)}{\longrightarrow} T_{V}(N) \rightarrow 0$. Thus we have a commutative diagram with exact rows

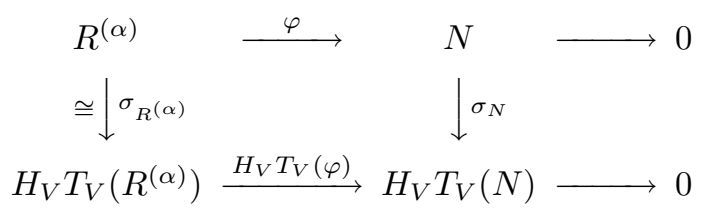

where $\sigma_{R^{(\alpha)}}$ is an isomorphism, since $V$ is selfsmall. This proves that $\sigma_{N}$ is epic, for any $N \in \operatorname{Mod}-R$. In order to prove that $\rho$ is monic in $\mathcal{A}$, thanks to statement a) in Proposition 1.1, it is sufficient to prove that $\rho$ is monic in Gen $V=\operatorname{Pres} V$. Moreover, we see that $\rho$ is monic in $T_{V}(\operatorname{Mod}-R)$, since by adjunction $\rho_{T_{V}(-)} \circ$ $T_{V}\left(\sigma_{-}\right)=1_{T_{V}(-)}$, and $T_{V}\left(\sigma_{-}\right)$is an isomorphism, since we have already proved that $\sigma_{-}$, and so $T_{V}\left(\sigma_{-}\right)$, is an epimorphism in Mod- $R$. Therefore, it remains to be proved that Pres $V \subseteq T_{V}$ (Mod- $R$ ). Let $M \in$ Pres $V$ and let $V^{(\beta)} \rightarrow V^{(\alpha)} \stackrel{\varphi}{\rightarrow} M \rightarrow 0$ be exact. Applying $T_{V}$ to the exact sequence

$$
H_{V}\left(V^{(\beta)}\right) \rightarrow H_{V}\left(V^{(\alpha)}\right) \stackrel{\text { Coker } H_{V}(\varphi)}{\longrightarrow} C \rightarrow 0
$$


we obtain the commutative diagram with exact rows

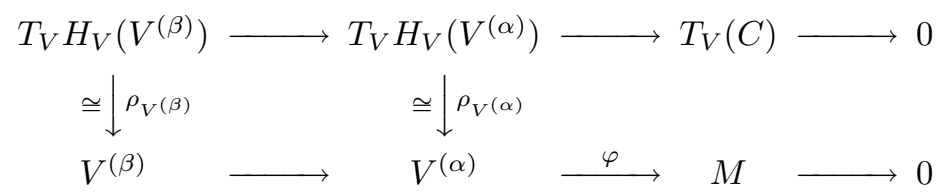

which proves that $M \cong T_{V}(C) \in T_{V}(\operatorname{Mod}-R)$.

(c) $\Rightarrow$ (a) This is an immediate consequence of Proposition 1.1 .

\section{THE TILTING THEOREM}

Here we shall obtain a tilting theorem in our present setting with the aid of

Lemma 3.1. Let $V \in \mathcal{A}$ be a tilting object, $R=\operatorname{End}_{\mathcal{A}}(V)$, and let $T_{V}^{(i)}, i \geq 1$, be the $i$-th left derived functor of $T_{V}$. Then:

a) Faith $V=\operatorname{Ker} T_{V}^{\prime}$;

b) $T_{V}^{(i)}=0$ for all $i \geq 2$;

c) $\mathrm{Ann}_{V}$ is an idempotent radical;

d) $\left(\operatorname{Ker} T_{V}, \operatorname{Ker} T_{V}^{\prime}\right)$ is a torsion theory in Mod- $R$;

e) for any $N \in \operatorname{Mod}-R$ the canonical inclusion $\operatorname{Ann}_{V}(N) \hookrightarrow N$ induces a natural isomorphism $T_{V}^{\prime}\left(\operatorname{Ann}_{V}(N)\right) \cong T_{V}^{\prime}(N)$.

Proof. a) If $N \in$ Faith $V$, then by d) and e) of Proposition 1.1 there is an exact sequence in Faith $V$

$$
0 \rightarrow K \rightarrow R^{(\alpha)} \rightarrow N \rightarrow 0 .
$$

On the one hand we have the exact sequence

$$
0 \rightarrow T_{V}^{\prime}(N) \rightarrow T_{V}(K) \rightarrow T_{V}\left(R^{(\alpha)}\right) \rightarrow T_{V}(N) \rightarrow 0,
$$

on the other hand, thanks to Theorem 2.4, we know that $V$ is a $*$-object, and so by Lemma 1.5 a) the functor $T_{V}$ preserves the exactness of sequences in Faith $V$. Thus $T_{V}^{\prime}(N)=0$, and the inclusion Faith $V \subseteq \operatorname{Ker} T_{V}^{\prime}$ is proved. Conversely, for any $N \in \operatorname{Ker} T_{V}^{\prime}$ we have a commutative diagram with exact rows

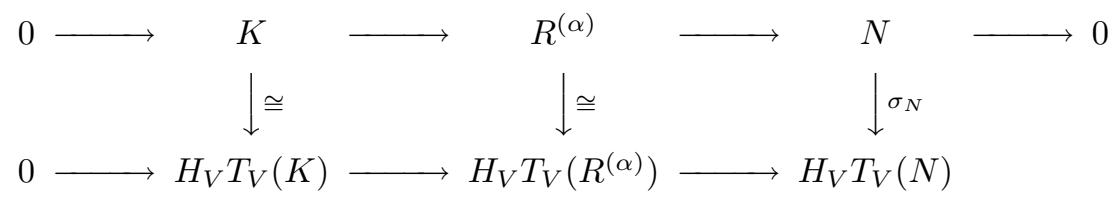

where the first two vertical canonical maps are isomorphisms thanks to Theorem 2.4. This shows that $\sigma_{N}$ is monic, so that $N \in$ Faith $V$ by statement c) in Proposition 1.1 .

b) Given any $N \in \operatorname{Mod}-R$ and short exact sequence

$$
0 \rightarrow K \rightarrow R^{(\alpha)} \rightarrow N \rightarrow 0,
$$

since $K \in$ Faith $V=\operatorname{Ker} T_{V}^{\prime}$, we see by induction that $T^{(i+1)}(N) \cong T^{(i)}(K)$ is zero for any $i \geq 1$.

c) We have already remarked in b) of Proposition 1.1 that $\mathrm{Ann}_{V}$ is a radical. Since by a) Faith $V=\operatorname{Ker} T_{V}^{\prime}$ is obviously closed under extensions, we can conclude that the associate radical $\mathrm{Ann}_{V}$ is idempotent.

d) Thanks to c) we see that $\left(\mathcal{T}, \operatorname{Ker} T_{V}^{\prime}\right)$ is a torsion theory, where $\mathcal{T}=\{N \in$ $\left.\operatorname{Mod}-R \mid \operatorname{Ann}_{V}(N)=N\right\}$. It remains to be proved that $\mathcal{T}=\operatorname{Ker} T_{V}$. First, let 
$N \in \mathcal{T}$. Then by a) in Proposition 1.1 we have $T_{V}(N) \cong T_{V}\left(N / \operatorname{Ann}_{V}(N)\right)=$ $T_{V}(0)=0$. Conversely, if $N \in \operatorname{Ker} T_{V}$, then for any embedding $L \hookrightarrow N$ we have $T_{V}(i)=0$, which proves that $\operatorname{Ann}_{V}(N)=N$, i.e., $N \in \mathcal{T}$.

e) Given any $N \in \operatorname{Mod}-R$ and the associated canonical exact sequence

$$
0 \rightarrow \operatorname{Ann}_{V}(N) \rightarrow N \rightarrow N / \operatorname{Ann}_{V}(N) \rightarrow 0,
$$

employing a), b) and d) we see that $T_{V}^{\prime}\left(\operatorname{Ann}_{V}(N)\right) \cong T_{V}^{\prime}(N)$ canonically.

Our Tilting Theorem follows. We note that several of the arguments are closely related to those in the proofs of various less general versions, but we include them for the sake of completeness.

Theorem 3.2. Let $V$ be a tilting object in an abelian category $\mathcal{A}, R=\operatorname{End}_{\mathcal{A}}(V)$, $H_{V}=\operatorname{Hom}_{\mathcal{A}}(V,-), H_{V}^{\prime}=\operatorname{Ext}_{\mathcal{A}}^{1}(V,-), T_{V}$ the left adjoint to $H_{V}$, and $T_{V}^{\prime}$ the first left derived functor of $T_{V}$. Set

$$
\mathcal{T}=\operatorname{Ker} H_{V}^{\prime}, \mathcal{F}=\operatorname{Ker} H_{V}, \mathcal{X}=\operatorname{Ker} T_{V}, \mathcal{Y}=\operatorname{Ker} T_{V}^{\prime} .
$$

Then:

a) $(\mathcal{T}, \mathcal{F})$ is a torsion theory in $\mathcal{A}$ with $\mathcal{T}=\operatorname{Gen} V$, and $(\mathcal{X}, \mathcal{Y})$ is a torsion theory in $\operatorname{Mod}-R$ with $\mathcal{Y}=$ Faith $V$;

b) the functors $H_{V} \uparrow_{\mathcal{T}}, T_{V} \uparrow_{\mathcal{Y}}, H_{V}^{\prime} \uparrow_{\mathcal{F}}, T_{V}^{\prime} \uparrow_{\mathcal{X}}$ are exact, and they induce a pair of category equivalences $\mathcal{T} \underset{T_{V}}{\stackrel{H_{V}}{\leftrightarrows}} \mathcal{Y}$ and $\mathcal{F} \underset{T_{V}^{\prime}}{\stackrel{H_{V}^{\prime}}{\leftrightarrows}} \mathcal{X}$

c) $T_{V} H_{V}^{\prime}=0=T_{V}^{\prime} H_{V}$ and $H_{V} T_{V}^{\prime}=0=H_{V}^{\prime} T_{V}$;

d) there are natural transformations $\theta$ and $\eta$ that, together with the adjoint transformations $\rho$ and $\sigma$, yield exact sequences

$$
0 \rightarrow T_{V} H_{V}(M) \stackrel{\rho_{M}}{\longrightarrow} M \stackrel{\eta_{M}}{\longrightarrow} T_{V}^{\prime} H_{V}^{\prime}(M) \rightarrow 0
$$

and

$$
0 \rightarrow H_{V}^{\prime} T_{V}^{\prime}(N) \stackrel{\theta_{N}}{\longrightarrow} N \stackrel{\sigma_{N}}{\longrightarrow} H_{V} T_{V}(N) \rightarrow 0
$$

for each $M \in \mathcal{A}$ and for each $N \in \operatorname{Mod}-R$.

Proof. Statement a) is contained in Proposition 2.1 and Lemma 3.1. The first part of $b$ ) regarding the exactness of the four restricted functors and the existence of the first equivalence is an immediate consequence of Theorem 2.4, Lemma 1.5. Proposition [2.1 ) and Lemma 3.1 b). Moreover, part of d) is contained in Theorem 2.4 and Proposition 1.1

In order to prove c), we start with an arbitrary object $M \in \mathcal{A}$ and a fixed associated short exact sequence

$$
0 \rightarrow M \rightarrow X_{0} \rightarrow X_{1} \rightarrow 0
$$

with $X_{0}$ and $X_{1}$ objects of $\operatorname{Gen}(V)=\mathcal{T}$. Applying $\operatorname{Hom}_{\mathcal{A}}(V,-)$, we obtain the exact sequence $H_{V}\left(X_{0}\right) \rightarrow H_{V}\left(X_{1}\right) \rightarrow H_{V}^{\prime}(M) \rightarrow H_{V}^{\prime}\left(X_{0}\right)=0$. Applying $T_{V}$ we obtain the commutative diagram with exact rows

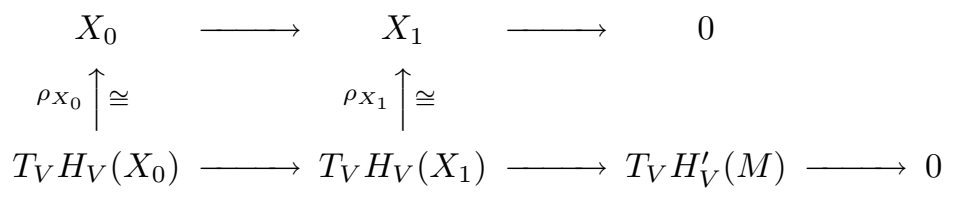

which shows that $T_{V} H_{V}^{\prime}(M)=0$. Moreover, thanks to Proposition 1.1d) and Lemma 3.17), we have $H_{V}(M) \in$ Faith $V=\operatorname{Ker} T_{V}^{\prime}$, and so $T_{V}^{\prime} H_{V}(M)=0$. 
On the other hand, for any $N \in \operatorname{Mod}-R$ let us consider an exact sequence of the form

$$
0 \rightarrow K \rightarrow R^{(\alpha)} \rightarrow N \rightarrow 0
$$

Note that both $R^{(\alpha)}$ and the submodule $K$ belong to Faith $V$. Applying $H_{V}$ to the exact sequence $0=T_{V}^{\prime}\left(R^{(\alpha)}\right) \rightarrow T_{V}^{\prime}(N) \rightarrow T_{V}(K) \rightarrow_{V}\left(R^{(\alpha)}\right)$, we obtain the commutative diagram with exact rows

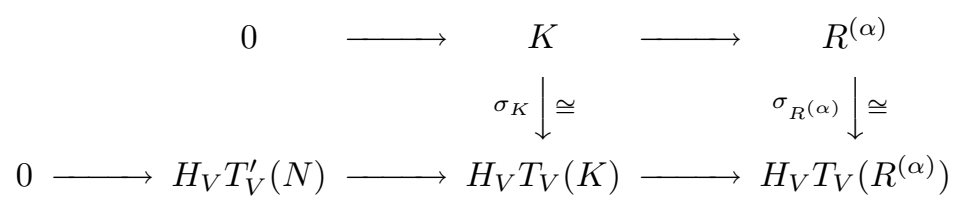

which shows that $H_{V} T_{V}^{\prime}(N)=0$. Finally, by Proposition $\left.1.1 \mathrm{~d}\right)$ and hypothesis, we have $T(N) \in \operatorname{Gen}(V)=\mathcal{T}=\operatorname{Ker} H_{V}^{\prime}$, therefore $H_{V}^{\prime} T_{V}(N)=0$. This completes the proof of $\mathrm{c}$ ).

In order to prove the second half of b), first we remark that the inclusion $\operatorname{Im} H_{V}^{\prime} \subseteq$ $\mathcal{X}$ follows from $T_{V} H_{V}^{\prime}=0$ and, similarly, the inclusion $\operatorname{Im} T_{V}^{\prime} \subseteq \mathcal{F}$ follows from $H_{V} T_{V}^{\prime}=0$.

Next, let $M \in \mathcal{F}$. Applying $\operatorname{Hom}_{\mathcal{A}}(V,-)$ to the exact sequence $(*)$, we obtain the exact sequence $0 \rightarrow H_{V}\left(X_{0}\right) \rightarrow H_{V}\left(X_{1}\right) \rightarrow H_{V}^{\prime}(M) \rightarrow 0$, and applying $T_{V}$ to this, we obtain the diagram with exact rows

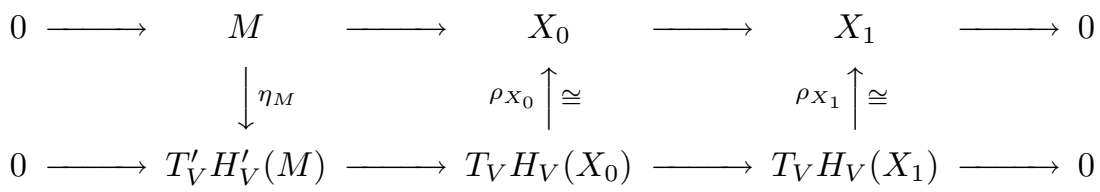

where $\eta_{M}$ is the unique isomorphism making the diagram commutative. Similarly, given any $N \in \mathcal{X}$ and any exact sequence of the form (**), we define $\theta_{N}: H_{V}^{\prime} T_{V}^{\prime}(N) \rightarrow N$ as the unique isomorphism making commutative the diagram

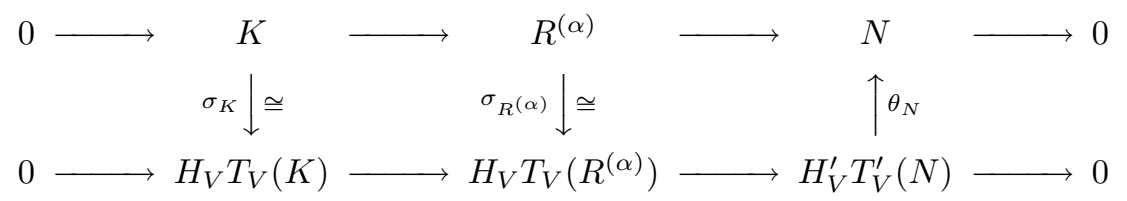

It can be shown that $\theta_{N}$ does not depend on the choice of $(* *)$, and that $\left(\eta_{M}\right)_{M \in \mathcal{F}}$ and $\left(\theta_{N}\right)_{N \in \mathcal{X}}$ are natural maps.

This proves that $\mathcal{F} \underset{T_{V}^{\prime}}{\stackrel{H_{V}^{\prime}}{\rightleftarrows}} \mathcal{X}$ is an equivalence.

To complete the proof of d), we first recall that Lemma 3.1p) says that for any $N \in \operatorname{Mod}-R$ the canonical inclusion $\operatorname{Ann}_{V}(N) \hookrightarrow N$ induces a natural isomorphism $T_{V}^{\prime}\left(\operatorname{Ann}_{V}(N)\right) \cong T_{V}^{\prime}(N)$. Second, since from Proposition 2.1k) we have $\operatorname{proj} \operatorname{dim} V \leq 1$, we can similarly prove that for any $M \in \mathcal{A}$ the canonical projection $M \rightarrow M / \operatorname{Tr}_{V}(M)$ induces a natural isomorphism $H_{V}^{\prime}(M) \cong H_{V}^{\prime}\left(M / \operatorname{Tr}_{V}(M)\right)$. 
Because of this, we can extend the definitions of $\eta$ and $\theta$ to a pair of natural homomorphisms defined in $\mathcal{A}$ and in Mod- $R$ respectively, making the diagrams

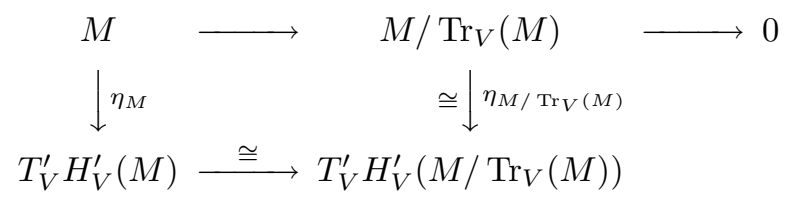

and

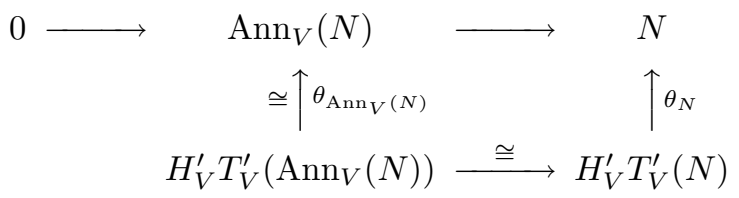

commutative for any $M \in \mathcal{A}$ and any $N \in \operatorname{Mod}-R$. Thus we see that $\eta_{M}$ is epic, $\operatorname{Ker}\left(\eta_{M}\right)=\operatorname{Tr}_{V}(M), \theta_{N}$ is monic and $\operatorname{Im}\left(\theta_{N}\right)=\operatorname{Ann}_{V}(N)$. Applying Proposition 1.1. $)$, we complete the proof of $\mathrm{d}$ ).

\section{REPRESENTING FAITHFUL TORSION THEORIES}

Given any abelian category $\mathcal{M}$, let us denote by $\mathcal{D}^{b}(\mathcal{M})$ the bounded derived category of $\mathcal{M}$. If $(\mathcal{X}, \mathcal{Y})$ is a torsion theory in $\mathcal{M}$, then $\mathcal{H}(\mathcal{X}, \mathcal{Y})$ is the full subcategory of $\mathcal{D}^{b}(\mathcal{M})$ defined as

$$
\mathcal{H}(\mathcal{X}, \mathcal{Y})=\left\{X \in \mathcal{D}^{b}(\mathcal{M}) \mid H^{-1}(X) \in \mathcal{Y}, H^{0}(X) \in \mathcal{X}, H^{i}(X)=0 \forall i \neq-1,0\right\} .
$$

$\mathcal{H}(\mathcal{X}, \mathcal{Y})$ is called the heart of the t-structure in $\mathcal{D}^{b}(\mathcal{M})$ associated with $(\mathcal{X}, \mathcal{Y})$.

Regarding a map $X^{-1} \stackrel{x}{\longrightarrow} X^{0}$ as a complex $\ldots 0 \rightarrow X^{-1} \stackrel{x}{\longrightarrow} X^{0} \rightarrow 0 \ldots$, the objects of $\mathcal{H}(\mathcal{X}, \mathcal{Y})$ are represented, up to isomorphism, by complexes of the form

$$
X: X^{-1} \stackrel{x}{\longrightarrow} X^{0} \text { with } \operatorname{Ker} x \in \mathcal{Y} \text { and } \operatorname{Coker} x \in \mathcal{X} .
$$

A morphism in $\mathcal{H}(\mathcal{X}, \mathcal{Y})$ is a formal fraction $\varphi=s^{-1} f$, where:

(1) $X \stackrel{f}{\longrightarrow} Y$ is a representative of a homotopy class of complex maps

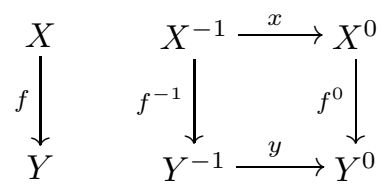

where $X \stackrel{f}{\longrightarrow} Y$ is null-homotopic if there is a map $r^{0}: X^{0} \rightarrow Y^{-1}$ such that

$$
f^{0}=y r^{0} \text { and } f^{-1}=r^{0} x
$$

(2) $X \stackrel{s}{\longrightarrow} Y$ is a quasi-isomorphism, i.e., there are isomorphisms making the diagrams

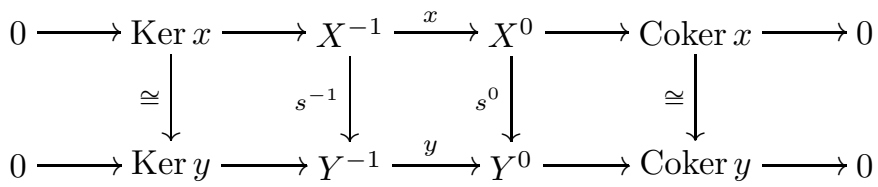

commute: quasi-isomorphisms are invertible in $\mathcal{H}(\mathcal{X}, \mathcal{Y})$. 
It turns out that $\mathcal{H}(\mathcal{X}, \mathcal{Y})$ is an abelian category, and setting

$$
\mathcal{T}=\mathcal{Y}[1]=\{Y \rightarrow 0 \mid Y \in \mathcal{Y}\} \text { and } \mathcal{F}=\mathcal{X}=\{0 \rightarrow X \mid X \in \mathcal{X}\}
$$

the pair $(\mathcal{T}, \mathcal{F})$ is a torsion theory in $\mathcal{H}(\mathcal{X}, \mathcal{Y})$ with category equivalences $\mathcal{T} \cong \mathcal{Y}$ and $\mathcal{F} \cong \mathcal{X}$.

An exhaustive description of $\mathcal{H}(\mathcal{X}, \mathcal{Y})$ is contained in [14, Chapter 1.

If $\mathcal{M}$ has products and coproducts with good behaviour, as in the case of Mod- $R$, then $\mathcal{H}(\mathcal{X}, \mathcal{Y})$ is cocomplete.

Lemma 4.1. Let $\mathcal{M}$ be a complete and cocomplete abelian category with exact coproducts, such that for any family of objects the canonical map from their coproduct to their product is monic. Then for any torsion theory $(\mathcal{X}, \mathcal{Y})$ in $\mathcal{M}$ the associated heart $\mathcal{H}(\mathcal{X}, \mathcal{Y})$ is cocomplete.

Proof. Let $\alpha$ be any cardinal. By hypothesis, the diagram

$$
\amalg: \prod_{\alpha} \mathcal{M} \rightleftarrows \mathcal{M}: \Delta,
$$

where $\amalg$ is the coproduct functor and $\Delta$ is the diagonal functor, defines an adjoint pair $\langle\amalg, \Delta\rangle$. This adjunction naturally extends componentwise to the corresponding homotopy categories. Moreover, since both $\amalg$ and $\Delta$ are exact, they extend to a pair of functors $\widehat{\amalg}$ and $\widehat{\Delta}$ between the corresponding derived categories. Moreover, thanks to [16], Section 3, the diagram

$$
\widehat{\amalg}: \mathcal{D}^{b}\left(\prod_{\alpha} \mathcal{M}\right) \cong \prod_{\alpha} \mathcal{D}^{b}(\mathcal{M}) \rightleftarrows \mathcal{D}^{b}(\mathcal{M}): \widehat{\Delta}
$$

still defines an adjoint pair $\langle\widehat{U}, \widehat{\Delta}\rangle$. This shows that $\mathcal{D}^{b}(\mathcal{M})$ admits arbitrary coproducts, and that they are defined componentwise. Moreover, since the assumptions on $\mathcal{M}$ guarantee that both $\mathcal{X}$ and $\mathcal{Y}$ are closed under arbitrary coproducts, we see that $\mathcal{H}(\mathcal{X}, \mathcal{Y})$ is closed under coproducts in $\mathcal{D}^{b}(\mathcal{M})$.

Thus by Theorem 1.4 we immediately have:

Proposition 4.2. If $(\mathcal{X}, \mathcal{Y})$ is a torsion theory in Mod- $R$ there is a $*$-object $V=$ $\left(R / \mathbf{r}_{R}(\mathcal{Y})\right)[1]$ in $\mathcal{H}(\mathcal{X}, \mathcal{Y})$ that induces an equivalence

$$
H_{V}: \mathcal{T} \rightleftarrows \mathcal{Y}: T_{V}
$$

Definition 4.3. A torsion theory $(\mathcal{X}, \mathcal{Y})$ in $\operatorname{Mod}-R$ is faithful if $\mathbf{r}_{R}(\mathcal{Y})=0$.

Note that $(\mathcal{X}, \mathcal{Y})$ is faithful if and only if $R_{R} \in \mathcal{Y}$ or, equivalently, if $\mathcal{Y}$ generates Mod- $R$.

We shall show that when $(\mathcal{X}, \mathcal{Y})$ is faithful in Mod- $R$, the equivalence $H_{V}$ : $\mathcal{T} \rightleftarrows \mathcal{Y}: T_{V}$ in Proposition 4.2 is actually induced by a tilting object $V$ with $\operatorname{End}_{\mathcal{H}}(V)=R$. To do so we need

Lemma 4.4. If $\mathcal{Y}$ generates $\mathcal{M}$, then every object of $\mathcal{H}(\mathcal{X}, \mathcal{Y})$ is isomorphic to a complex of the form $Y^{-1} \rightarrow Y^{0}$, with $Y^{-1}, Y^{0} \in \mathcal{Y}$.

Proof. Let $Z^{-1} \stackrel{z}{\longrightarrow} Z^{0} \in \mathcal{H}(\mathcal{X}, \mathcal{Y})$ to obtain exact sequences

$$
0 \rightarrow Y \rightarrow Z^{-1} \rightarrow I \rightarrow 0 \text { and } 0 \rightarrow I \rightarrow Z^{0} \rightarrow X \rightarrow 0
$$


with $I=\operatorname{Im} z, Y \in \mathcal{Y}$ and $X \in \mathcal{X}$. Then there are an object $Y^{0} \in \mathcal{Y}$, an epimorphism $Y^{0} \rightarrow Z^{0}$ and a pullback diagram

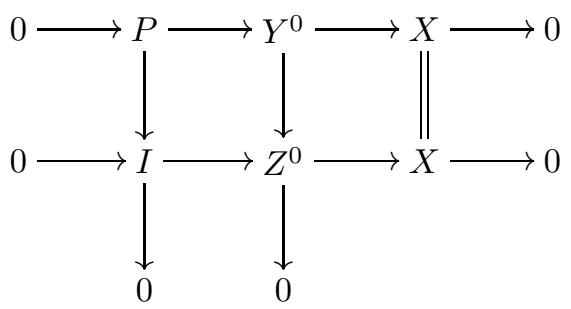

where $P$ is in $\mathcal{Y}$, since $\mathcal{Y}$ is closed under subobjects. Then we obtain a further pullback diagram

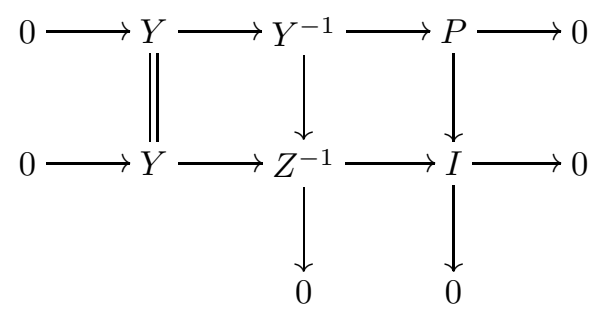

where $Y^{-1}$ is in $\mathcal{Y}$, since $\mathcal{Y}$ is closed under extensions. Now (1) and (2) combine to give a commutative diagram with exact rows

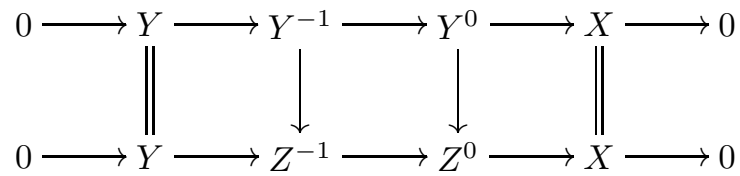

and so the desired quasi-isomorphism.

This allows us to prove the following version of Proposition 3.2(ii) on page 17 of [14.

Proposition 4.5. If $\mathcal{Y}$ generates $\mathcal{M}$, then $\mathcal{T}=\mathcal{Y}[1]$ cogenerates $\mathcal{H}(\mathcal{X}, \mathcal{Y})$.

Proof. By the last lemma, we know that every object in $\mathcal{H}(\mathcal{X}, \mathcal{Y})$ is isomorphic to a complex of the form $Y^{-1} \stackrel{y}{\rightarrow} Y^{0}$ with $Y^{-1}, Y^{0} \in \mathcal{Y}$. We shall show that

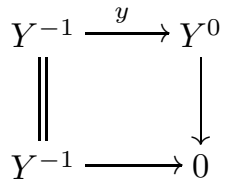

is a monomorphism. So suppose that the commutative diagram

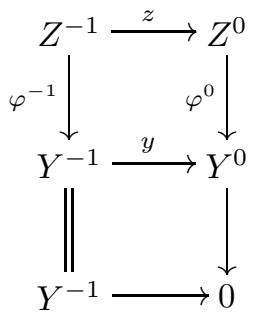


yields a null-homotopic map, i.e., that there is a map $r^{0}: Z^{0} \rightarrow Y^{-1}$ such that

$$
\varphi^{-1}=r^{0} z \text {. }
$$

Let $\gamma=y r^{0}-\varphi^{0}: Z^{0} \rightarrow Y^{0}$ so that

$$
\gamma z=y r^{0} z-\varphi^{0} z=y \varphi^{-1}-y \varphi^{-1}=0
$$

and hence $\operatorname{Im} z \subseteq \operatorname{Ker} \gamma$. But $Z^{0} / \operatorname{Im} z \in \mathcal{X}$ and $Z^{0} / \operatorname{Ker} \gamma \in \mathcal{Y}$. Thus $\gamma=0$ and so

$$
\varphi^{0}=y r^{0} \text {. }
$$

In other words the map

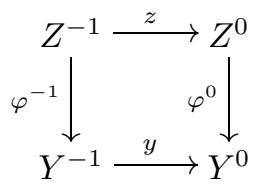

is zero in $\mathcal{H}(\mathcal{X}, \mathcal{Y})$, which proves our assertion.

Now we have the needed results to prove

Theorem 4.6. A torsion theory $(\mathcal{X}, \mathcal{Y})$ in $\operatorname{Mod}-R$ is faithful if and only if there is a cocomplete abelian category $\mathcal{H}$ and a tilting object $V$ of $\mathcal{H}$ such that $R=\operatorname{End}_{\mathcal{H}}(V)$ and $\mathcal{Y}=$ Faith $V$.

Proof. The condition is sufficient by Theorem 3.2. Necessity follows from Propositions 4.2 and 4.5 , and from Theorem 2.4 .

\section{The hereditary CASE}

Throughout this section $\mathcal{A}$ is a fixed hereditary cocomplete abelian category, $V$ is a tilting object in $\mathcal{A}$ with $R=\operatorname{End}_{\mathcal{A}}(V)$, and $(\mathcal{T}, \mathcal{F}),(\mathcal{X}, \mathcal{Y})$ are the induced torsion theories in $\mathcal{A}$ and Mod- $R$, respectively. Here we shall show that $R$ is quasitilted, and verify that it satisfies key properties of quasitilted algebras.

Lemma 5.1. $\operatorname{proj} \operatorname{dim} N \leq 1$ for any $N \in \mathcal{Y}$.

Proof. Let $N \in \mathcal{Y}$ and consider an exact sequence in Mod- $R$,

$$
0 \rightarrow K \rightarrow P \rightarrow N \rightarrow 0
$$

with $P$ projective. Since this sequence is exact in $\mathcal{Y}$ we have

$$
0 \rightarrow T_{V}(K) \rightarrow T_{V}(P) \rightarrow T_{V}(N) \rightarrow 0
$$

exact in $\mathcal{T}$. Since $\mathcal{T}=$ Pres $V$, there is an exact sequence

$$
0 \rightarrow L \rightarrow V^{(\alpha)} \rightarrow T_{V}(K) \rightarrow 0
$$

in $\mathcal{T}$. Now apply $\operatorname{Hom}_{\mathcal{A}}(-, L)$ to $(*)$ to obtain

$$
\operatorname{Ext}_{\mathcal{A}}^{1}\left(T_{V}(P), L\right) \rightarrow \operatorname{Ext}_{\mathcal{A}}^{1}\left(T_{V}(K), L\right) \rightarrow 0=\operatorname{Ext}_{\mathcal{A}}^{2}\left(T_{V}(N), L\right) .
$$

But, since $T_{V}(P) \in$ Add $V$ and $L \in \mathcal{T}, \operatorname{Ext}_{\mathcal{A}}^{1}\left(T_{V}(P), L\right)=0$ (see Corollary 8.3 ), and hence $\operatorname{Ext}_{\mathcal{A}}^{1}\left(T_{V}(K), L\right)=0$. Thus $(* *)$ splits, so $T_{V}(K) \in \operatorname{Add} V$ and $K \cong$ $H_{V} T_{V}(K)$ is projective.

Proposition 5.2. The torsion theory $(\mathcal{X}, \mathcal{Y})$ splits in $\operatorname{Mod}-R$. 
Proof. Let $A \in \mathcal{T}$ and $B \in \mathcal{F}$. Then, since $\overline{\mathrm{Gen}} V=\mathcal{A}$, there is an exact sequence

$$
0 \rightarrow B \stackrel{f}{\rightarrow} A_{1} \stackrel{g}{\rightarrow} A_{2} \rightarrow 0
$$

with $A_{1}, A_{2} \in \mathcal{T}$. Applying $H=H_{V}$ we obtain an exact sequence

$$
0 \rightarrow H A_{1} \stackrel{H g}{\longrightarrow} H A_{2} \stackrel{\partial}{\rightarrow} H^{\prime} B \rightarrow 0
$$

since $H^{\prime} A_{1}=0$. Now, from these two exact sequences we obtain a commutative diagram

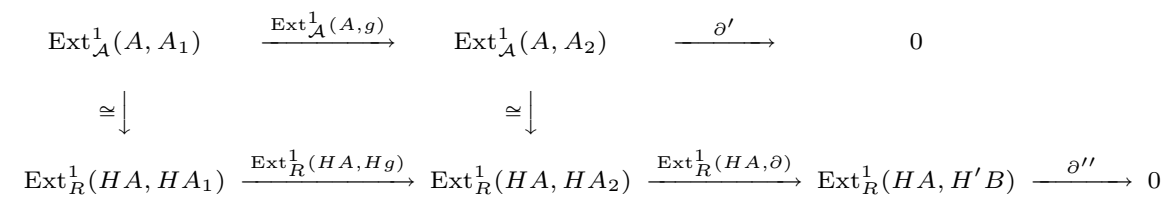

with exact rows, noting that $\partial^{\prime}=0$ since $\mathcal{A}$ is hereditary, and $\partial^{\prime \prime}=0$ by Lemma 5.1. Also, the vertical maps are isomorphisms, since $\mathcal{T}$ and $\mathcal{Y}$ are closed under extensions, and $H_{V}$ is exact on $\mathcal{T}$ and $T_{V}$ is exact on $\mathcal{Y}$. Thus $\operatorname{Ext}_{R}^{1}\left(H A, H^{\prime} B\right)=0$, and so the exact sequence

$$
0 \rightarrow H_{V}^{\prime} T_{V}^{\prime}(N) \stackrel{\theta_{N}}{\longrightarrow} N \stackrel{\sigma_{N}}{\longrightarrow} H_{V} T_{V}(N) \rightarrow 0
$$

splits for all $N \in \operatorname{Mod}-R$.

Thus, using the Tilting Theorem, Theorem [3.2, we have shown that $R$ is a quasitilted ring. We shall conclude this section by showing that $R$ enjoys two further properties possessed by the quasitilted algebras of [14.

Proposition 5.3. $\mathrm{rtgl} \operatorname{dim} R \leq 2$.

Proof. Suppose

$$
0 \rightarrow K \rightarrow P_{1} \stackrel{d}{\rightarrow} P_{0} \rightarrow M \rightarrow 0
$$

exact with $P_{0}$ and $P_{1}$ projective in $\operatorname{Mod}-R$. Let $I=\operatorname{Im} d$, so that

$$
0 \rightarrow K \rightarrow P_{1} \rightarrow I \rightarrow 0
$$

is exact in $\mathcal{Y}$. Now apply Lemma 5.1

Note that this last argument can be modified to show that an arbitrary ring $R$ with faithful torsion theory $(\mathcal{X}, \mathcal{Y})$ in $\operatorname{Mod}-R$ satisfies

$$
\text { rt gl } \operatorname{dim} R \leq \operatorname{proj} \operatorname{dim} \mathcal{Y}+1 \text {. }
$$

Proposition 5.4. $\operatorname{inj} \operatorname{dim} N \leq 1$ for any $N \in \mathcal{X}$.

Proof. If $N \in \mathcal{X}$, then so is $\mathrm{E}(N)$, since from Proposition 5.2 we know that the torsion theory $(\mathcal{X}, \mathcal{Y})$ splits. Thus there is an exact sequence in $\mathcal{X}$

$$
0 \rightarrow N \stackrel{d_{0}}{\longrightarrow} E_{0} \stackrel{d_{1}}{\longrightarrow} E_{1} \stackrel{d}{\rightarrow} C \rightarrow 0
$$

in which each $\operatorname{Im} d_{i}$ is essential in the injective module $E_{i}$ and

$$
\left(T_{V}^{\prime} K\right) \quad 0 \rightarrow T_{V}^{\prime}(N) \stackrel{T_{V}^{\prime}\left(d_{0}\right)}{\longrightarrow} T_{V}^{\prime}\left(E_{0}\right) \stackrel{T_{V}^{\prime}\left(d_{1}\right)}{\longrightarrow} T_{V}^{\prime}\left(E_{1}\right) \stackrel{T_{V}^{\prime}(d)}{\longrightarrow} T_{V}^{\prime}(C) \rightarrow 0
$$


represents the zero element in $\operatorname{Ext}_{\mathcal{A}}^{2}\left(T_{V}^{\prime}(C), T_{V}^{\prime}(N)\right)$. Let

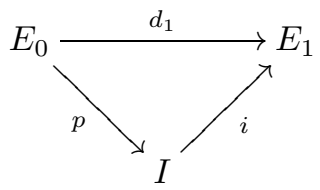

be the epi-monic factorization through $I=\operatorname{Im} d_{1} \in \mathcal{X}$, to obtain short exact sequences

$\left(T_{V}^{\prime} E\right) \quad 0 \rightarrow T_{V}^{\prime}(N) \stackrel{T_{V}^{\prime}\left(d_{0}\right)}{\longrightarrow} T_{V}^{\prime}\left(E_{0}\right) \stackrel{T_{V}^{\prime}(p)}{\longrightarrow} T_{V}^{\prime}(I) \rightarrow 0$

and

$\left(T_{V}^{\prime} F\right)$

$0 \rightarrow T_{V}^{\prime}(I) \stackrel{T_{V}^{\prime}(i)}{\longrightarrow} T_{V}^{\prime}\left(E_{1}\right) \stackrel{T_{V}^{\prime}(d)}{\longrightarrow} T_{V}^{\prime}(C) \rightarrow 0$

then, according to 18, page 175, Lemma 4.1, $T_{V}^{\prime} K \sim 0$ if and only if there is a short exact sequence $L$ in $\mathcal{A}$ such that $T_{V}^{\prime} F \sim T_{V}^{\prime}(p) L$ and $T_{V}^{\prime} E T_{V}^{\prime}(p) \sim 0$. In particular the first condition gives a commutative diagram with exact rows

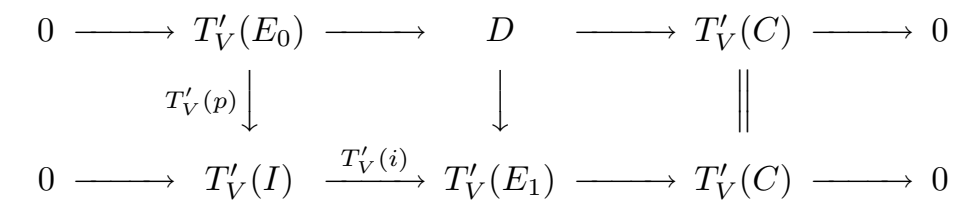

$\left(T_{V}^{\prime} F\right) \quad 0 \longrightarrow T_{V}^{\prime}(I) \stackrel{T_{V}^{\prime}(i)}{\longrightarrow} T_{V}^{\prime}\left(E_{1}\right) \longrightarrow 0$

Now, since $\mathcal{F}$ is closed under extensions, all the previous diagrams belong to $\mathcal{F}$, and so we can apply $H_{V}^{\prime}$ to see that there is a commutative diagram with exact rows in Mod- $R$

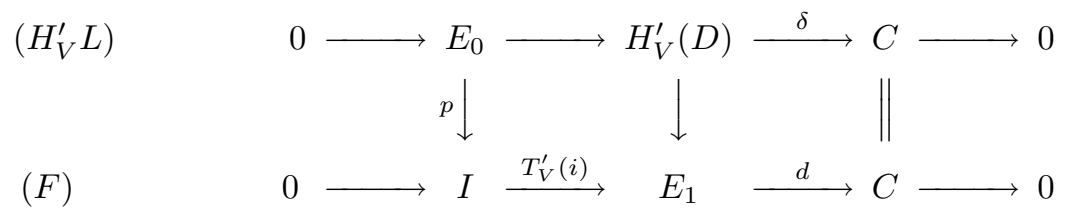

in which, since $E_{0}$ is injective, $\delta$ is epi-split, and so $d$ is such. Thus $F$ splits, $I$ is injective, and

$$
0 \rightarrow N \stackrel{d_{0}}{\rightarrow} E_{0} \stackrel{p}{\rightarrow} I \rightarrow 0
$$

is an injective resolution of $N$.

\section{QuAsitilted RINGS CHARACTERIZED}

We reiterate from the Introduction:

Definition 6.1. A ring $R$ is called a right quasitilted ring if there is a faithful splitting torsion theory $(\mathcal{X}, \mathcal{Y})$ in $\operatorname{Mod}-R$ such that proj $\operatorname{dim} \mathcal{Y} \leq 1$.

The results from Section 5 can be summarized in the following.

Proposition 6.2. If $V$ is a tilting object in a hereditary cocomplete abelian category $\mathcal{A}$, then $R=\operatorname{End}_{\mathcal{A}}(V)$ is a quasitilted ring with $\mathcal{X}=\operatorname{Ker} T_{V}$ and $\mathcal{Y}=$ Faith $V$. Moreover $\operatorname{inj} \operatorname{dim} \mathcal{X} \leq 1$ and $\operatorname{rtgl} \operatorname{dim} R \leq 2$.

This section is devoted to proving the converse of Proposition 6.2 To do so, we need one more lemma. 
Lemma 6.3. Let $V$ be a tilting object in an abelian category $\mathcal{A}$, and let $R=$ End $_{\mathcal{A}}(V)$. Then for all $L, M \in \mathcal{T}=$ Gen $V$ and for all $i \geq 0$,

$$
\operatorname{Ext}_{R}^{i}\left(H_{V}(L), H_{V}(M)\right) \cong \operatorname{Ext}_{\mathcal{A}}^{i}(L, M) \text {. }
$$

Proof. The same proof as in [6], Lemma 3.6.2, works in this general setting.

Theorem 6.4. A ring $R$ is right quasitilted via the torsion theory $(\mathcal{X}, \mathcal{Y})$ if and only if there exist a hereditary cocomplete abelian category $\mathcal{A}$ and a tilting object $V$ in $\mathcal{A}$ such that $R \cong \operatorname{End}_{\mathcal{A}}(V)$. Moreover in this case $\operatorname{inj} \operatorname{dim} \mathcal{X} \leq 1$ and $\mathrm{rtgl} \operatorname{dim} R \leq 2$.

Proof. Thanks to Proposition 6.2 it remains to be proved that any quasitilted ring $R$ is isomorphic to $\operatorname{End}_{\mathcal{A}}(V)$ for a tilting object in a suitable hereditary cocomplete abelian category. Now let $\mathcal{H}, V$ and $(\mathcal{T}, \mathcal{F})$ as in Theorem 4.6. To finish the proof, we have to show that $\mathcal{H}$ is hereditary. First, since by Proposition 4.5 any object $M$ in $\mathcal{H}$ admits an exact sequence

$$
0 \rightarrow M \rightarrow X_{0} \rightarrow X_{1} \rightarrow 0
$$

with $X_{0}, X_{1} \in \mathcal{T}$, for any $L \in \mathcal{H}$ we have an exact sequence

$$
\operatorname{Ext}_{\mathcal{H}}^{2}\left(X_{0}, L\right) \rightarrow \operatorname{Ext}_{\mathcal{H}}^{2}(M, L) \rightarrow \operatorname{Ext}_{\mathcal{H}}^{3}\left(X_{1}, L\right)
$$

Therefore it is enough to prove that $\operatorname{Ext}_{\mathcal{H}}^{2}(\mathcal{T}, \mathcal{H})=0$ (from which it follows easily from [18, Lemma 4.1, page 75, that even $\operatorname{Ext}_{\mathcal{H}}^{3}(\mathcal{T}, \mathcal{H})=0$ ) in order to see that $\operatorname{Ext}_{\mathcal{H}}^{2}(\mathcal{H}, \mathcal{H})=0$. Moreover, since $(\mathcal{T}, \mathcal{F})$ is a torsion theory in $\mathcal{H}$, we see that $\operatorname{Ext}_{\mathcal{H}}^{2}(\mathcal{T}, \mathcal{H})=0$ if and only if $\operatorname{Ext}_{\mathcal{H}}^{2}(\mathcal{T}, \mathcal{T})=0$ and $\operatorname{Ext}_{\mathcal{H}}^{2}(\mathcal{T}, \mathcal{F})=0$. The first Ext-vanishing is an immediate consequence of Lemma 6.3 in the case of $i=2$, since proj $\operatorname{dim} \mathcal{Y} \leq 1$ by assumption. In order to prove the second Ext-vanishing, let us consider $L \in \mathcal{T}$ and $M \in \mathcal{F}$. Given an exact sequence $\left.{ }^{*}\right)$ for $M$, applying $H=H_{V}$, since $H M=0$ and $H^{\prime} X_{0}=0$, we obtain an exact sequence

$$
0 \rightarrow H X_{0} \rightarrow H X_{1} \rightarrow H^{\prime} M \rightarrow 0,
$$

and from $(*)$ and $(* *)$ we obtain a commutative diagram with exact rows

$$
\begin{aligned}
& \operatorname{Ext}_{\mathcal{H}}^{1}\left(L, X_{0}\right) \longrightarrow \operatorname{Ext}_{\mathcal{H}}^{1}\left(L, X_{1}\right) \longrightarrow \operatorname{Ext}_{\mathcal{H}}^{2}(L, M) \longrightarrow \operatorname{Ext}_{\mathcal{H}}^{2}\left(L, X_{0}\right) \\
& \operatorname{Ext}_{R}^{1}\left(H L, H X_{0}\right) \longrightarrow \operatorname{Ext}_{R}^{1}\left(H L, H X_{1}\right) \longrightarrow \operatorname{Ext}_{R}^{1}\left(H L, H^{\prime} M\right)
\end{aligned}
$$

where $\operatorname{Ext}_{\mathcal{H}}^{2}\left(L, X_{0}\right)=0$ since $L$ and $X_{0}$ belong to $\mathcal{T}$, and $\operatorname{Ext}_{R}^{1}\left(H L, H^{\prime} M\right)=0$ since $H L \in \mathcal{Y}, H^{\prime} M \in \mathcal{X}$ and $(\mathcal{X}, \mathcal{Y})$ splits by assumption. Thus $\operatorname{Ext}_{\mathcal{H}}^{2}(L, M)=0$, and the proof is complete.

\section{An EXAmple AND TWO QUeStions}

Following the artin algebra tradition, we say that a ring $R$ is right tilted if there is a right hereditary ring $S$ with a finitely generated tilting module $V_{S}$ such that $R=\operatorname{End}\left(V_{S}\right)$ (see 4 for noetherian examples of such rings). Now, Theorem 6.4 shows that tilted rings are particular cases of quasitilted rings. In this section we will see that the class of (right) quasitilted rings properly extends the class of (right) tilted rings, and we shall discuss two problems that arise in connection with quasitilted algebras.

In the following,

$$
R=\left[\begin{array}{ll}
\mathbb{Q} & \mathbb{Q} \\
0 & \mathbb{Z}
\end{array}\right]
$$


denotes the ring of upper triangular $2 \times 2$ matrices over $\mathbb{Q}$ with 2,2 -entries in $\mathbb{Z}$. We let

$$
e=\left[\begin{array}{ll}
1 & 0 \\
0 & 0
\end{array}\right] \text { and } f=\left[\begin{array}{ll}
0 & 0 \\
0 & 1
\end{array}\right]
$$

in $R$, and we note that if $J=J(R)$, then

$$
f R e=0, \quad f R=f R f \cong \mathbb{Z}, \quad e R e \cong \mathbb{Q}, \quad e R f=e J=J \cong \mathbb{Q}_{\mathbb{Z}} .
$$

We shall show that $R$ is right quasitilted.

The ring $R$ is left, but not right hereditary, as observed by L. Small in [21]. Indeed according to a well-known result from [12]

$$
\text { rt } g l \operatorname{dim} R \leq \mathrm{rtgl} \operatorname{dim} e R e+\mathrm{rtgl} \operatorname{dim} f R f+1=2 .
$$

However there is an exact sequence

$$
0 \rightarrow K \longrightarrow f R^{(\alpha)} \longrightarrow e R \longrightarrow e R / e J \rightarrow 0,
$$

so we see that proj $\operatorname{dim} e R / e J=2$.

To prove that $R$ is quasitilted, we shall employ the following lemmas.

Lemma 7.1. All direct sums of copies of eR/eJ and of eR are injective.

Proof. To see that $e R / e J$ is injective, let $I \leq R_{R}$ and $\gamma: I \rightarrow e R / e J$. If $I \leq R f$, then $\gamma=0$. Otherwise $e \in I=e R+I f$, and one can show that Baer's Criterion applies.

Next we will show that $e R$ is injective relative to both $f R$ and $e R$, so [1, Proposition 16.12, applies. The former follows since $J=e R f_{f R f} \cong \mathbb{Q}_{\mathbb{Z}}$ and $f R=f R f_{f R f} \cong \mathbb{Z}_{\mathbb{Z}}$. For the latter, suppose that $I<e R, \gamma: I \rightarrow e R$. Then $I=e I f$ and again $\gamma(I) \leq e R f$ which is injective over $f R f$. Thus there is a map $\bar{\gamma}: e R f \rightarrow e R f$ that extends $\gamma$. Identifying $e R f=\mathbb{Q}_{\mathbb{Z}}$ we see that there is a $x \in \mathbb{Q}$ such that $\bar{\gamma}(e r f)=x e r f$ for all erf $\in e R f$. Now multiplication by $x e \in e R e \cong \operatorname{End}\left(e R_{R}\right)$ extends $\gamma$.

Clearly $e_{e} e R$ and $e R / e J$ have the d.c.c. on submodules, and in particular on annihilators of subsets of $R$. Thus (see 11, page 181) $R$ has a.c.c. on annihilators of subsets of $e R$ and $e R / e J$. Now, since $e R / e J$ and $e R$ are injective, the result follows from [11, Proposition 3, page 184.

Let $\mathcal{C}=\{e R / K \mid 0 \neq K \leq e R\}$ and let $(\mathcal{X}, \mathcal{Y})$ be the torsion theory generated by $\mathcal{C}$. Thus, letting

$$
\mathcal{Y}=\left\{Y_{R} \mid \operatorname{Hom}_{R}(C, Y)=0 \text { for all } C \in \mathcal{C}\right\}
$$

we have

$$
\mathcal{X}=\left\{X_{R} \mid \operatorname{Hom}_{R}(X, Y)=0 \text { for all } Y \in \mathcal{Y}\right\} .
$$

Lemma 7.2. $\mathcal{Y}=\left\{e R^{(\alpha)} \oplus N \mid N=N f\right\}$.

Proof. Let $Y \in \mathcal{Y}$. Since $\operatorname{Hom}_{R}(e R / K, Y)=0$ whenever $0 \neq K \leq e R$, it follows for $x \in Y$ that $x e \neq 0$ implies $x e R \cong e R$. Thus,

$$
Y=\sum_{I} w_{\alpha} e R+\sum_{L} b_{\lambda} f R
$$

with each $w_{\alpha} e R \cong e R$. Now let $H \subseteq I$ be maximal with $\left\{w_{\alpha} e R \mid \alpha \in H\right\}$ independent, so that $P=\bigoplus_{H} w_{\alpha} e R \cong e R^{(H)}$ is an (injective by Lemma 7.1) projective direct summand of $\sum_{I} w_{\alpha} e R$. One easily checks that $Y \cong e R^{(H)} \oplus N$ with $N=N f$. 
Suppose that $M=e R^{(\alpha)} \oplus N$ with $N=N f$. If $0 \neq \gamma \in \operatorname{Hom}_{R}(e R / K, M)$, then $\operatorname{Im} \gamma \subseteq e R^{(\alpha)}$ and $\operatorname{Im} \gamma \nsubseteq e J^{(\alpha)}=e J^{(\alpha)} f$, and so some $\pi_{\alpha} \gamma: e R / K \rightarrow e R$ is a split epimorphism. Thus $K=0$ and $M \in \mathcal{Y}$.

Corollary 7.3. $R \in \mathcal{Y}$ and $\operatorname{proj} \operatorname{dim} \mathcal{Y} \leq 1$.

Proof. Clearly $R \in \mathcal{Y}$, and proj $\operatorname{dim}\left(e R^{(\alpha)} \oplus N f\right) \leq 1$ since $e R^{(\alpha)}$ is projective and $\operatorname{proj} \operatorname{dim} N f \leq 1$ as it is an $f R=f R f \cong \mathbb{Z}$-module.

It only remains to show that $(\mathcal{X}, \mathcal{Y})$ splits. To do so we need

Lemma 7.4. $\mathcal{X}=$ Gen $\mathcal{C}$.

Proof. Clearly Gen $\mathcal{C} \subseteq \mathcal{X}$. So let $X \in \mathcal{X}$ and consider $X / X J$. Since every direct sum of copies of $e R / e J$ is injective by Lemma 7.1 as in the proof of Lemma 7.2, $X / X J \cong e R / e J^{(\alpha)} \oplus N$ with $N=N f$. But then $N \in \mathcal{X} \cap \mathcal{Y}=0$. Thus, since $J$ is nilpotent, there exist $t_{\alpha} \in X \backslash X J$ such that $\sum t_{\alpha} e R=X$, and by Lemmas 7.1 and 7.2, each $t_{\alpha} e R \cong e R / K_{\alpha}$ with $K_{\alpha} \neq 0$.

Proposition 7.5. $R$ is right quasitilted with torsion theory $(\mathcal{X}, \mathcal{Y})$.

Proof. If $X \in \mathcal{X}$ and (see Lemma 7.2) $Y=e R^{(\alpha)} \oplus N f$, then $\operatorname{Ext}_{R}^{1}(Y, X)=$ $\operatorname{Ext}_{R}^{1}(N f, X)$. To show that the latter is 0 , noting that by Lemma $7.4 \mathcal{X} \subseteq$ Gen $e R$, we will actually show that $\operatorname{Ext}_{R}^{1}(N, G)=0$ for any $G \in \operatorname{Gen} e R$ and $N=N f$ in $\operatorname{Mod}-R$. So suppose that

$$
0 \rightarrow K \longrightarrow e R^{(\alpha)} \longrightarrow G \rightarrow 0
$$

is exact, to obtain an exact sequence

$$
0=\operatorname{Ext}_{R}^{1}\left(N, e R^{(\alpha)}\right) \longrightarrow \operatorname{Ext}_{R}^{1}(N, G) \longrightarrow \operatorname{Ext}_{R}^{2}(N, K)=0 .
$$

Here the first equality is by Lemma 7.1 and the second is because $N=N f$ has projective dimension $\leq 1$.

Now, if $R$ is any right tilted ring with torsion theory $(\mathcal{X}, \mathcal{Y})$ in $\operatorname{Mod}-R$, then there is a right hereditary ring $S$ with a (finitely generated) tilting module $V_{S}$ such that $R=\operatorname{End}\left(V_{S}\right)$ and $\mathcal{X}=\operatorname{Ker}\left(-\otimes_{R} V\right)$. In any case if $V_{S}$ is a tilting module with $R=\operatorname{End}\left(V_{S}\right)$, then ${ }_{R} V$ is a tilting module and so is finitely presented, so that $\operatorname{Ker}\left(-\otimes_{R} V\right)$ is closed under direct products. As pointed out to us by Enrico Gregorio, the torsion theory $(\mathcal{X}, \mathcal{Y})$ of Proposition 7.5 cannot result from any tilting module, because the torsion-free injective module $e R$ embeds as a direct summand in $\prod_{0 \neq K \leq e R} e R / K$, so $\mathcal{X}$ is not closed under direct products. In a forthcoming article with Gregorio, we shall prove that $R$ is actually not a tilted ring.

A quasitilted artin algebra in the sense of Happel, Reiten and Smal $\varnothing$ [14 is one that has a split torsion theory $\left(\mathcal{X}_{0}, \mathcal{Y}_{0}\right)$ in $\bmod -R$ such that proj $\operatorname{dim} \mathcal{Y}_{0} \leq 1$ and $R \in \mathcal{Y}_{0}$, and, necessarily, $\operatorname{inj} \operatorname{dim} X_{0} \leq 1$. Clearly a quasitilted ring that happens to be an artin algebra is quasitilted in their sense. We wonder if the converse is true, and we shall next present some observations that suggest that it may be true.

Let $R$ be a quasitilted artin algebra with torsion theory $\left(\mathcal{X}_{0}, \mathcal{Y}_{0}\right)$ in $\bmod -R$, and let $(\mathcal{X}, \mathcal{Y})$ be the torsion theory in Mod- $R$ generated by $\mathcal{X}_{0}$. Then, according to [22], Proposition 2.5, page 140,

$\mathcal{X}=\left\{X \in \operatorname{Mod}-R \mid\right.$ non-zero factors of $X$ have non-zero submodules in $\left.\mathcal{X}_{0}\right\}$.

Claim 7.6. $\operatorname{inj} \operatorname{dim} \mathcal{X} \leq 1$. 
Proof. If $X \in \mathcal{X}$ there is a non-zero submodule $X_{0} \leq X$ with $\operatorname{inj} \operatorname{dim} X_{0} \leq 1$, so that given any simple module $S_{R}$ we have $\operatorname{Ext}_{R}^{2}\left(S, X_{0}\right)=0$. But then we have $X_{1} / X_{0} \leq X / X_{0}$ with $\operatorname{Ext}_{R}^{2}\left(S, X_{1} / X_{0}\right)=0$, and so $\operatorname{Ext}_{R}^{2}\left(S, X_{1}\right)=0$. Continue this way transfinitely to see that $X$ is a direct limit of modules of injective dimension $\leq 1$, and use [10], Lemma 3.1.16, to get $\operatorname{Ext}_{R}^{2}(S, X)=0$ for each simple $S_{R}$. But then for any $M \in \operatorname{Mod}-R$, considering the Loewy series of $M$, we have $\operatorname{Ext}_{R}^{2}(M, X)=0$. Thus inj $\operatorname{dim} \mathcal{X} \leq 1$.

\section{Now}

$\mathcal{Y}=\left\{Y_{R} \mid \operatorname{Hom}_{R}\left(\mathcal{X}_{0}, Y\right)=0\right\}=\left\{Y_{R} \mid\right.$ every fin. gen. submodule of $Y$ is in $\left.\mathcal{Y}_{0}\right\}$,

and, of course, $R_{R} \in \mathcal{Y}$.

Claim 7.7. proj $\operatorname{dim} \mathcal{Y} \leq 1$.

Proof. To show that $Y \in \mathcal{Y}$ has proj $\operatorname{dim} Y \leq 1$, consider an exact sequence

$$
0 \rightarrow K \rightarrow P \stackrel{f}{\rightarrow} Y \rightarrow 0
$$

where $P$ is projective. Since $R$ is semiperfect, $P=\bigoplus_{I} P_{\alpha}$, where each $P_{\alpha}$ is finitely generated. Now, for each finite subset $F \subseteq I$, let $K_{F}=\operatorname{Ker} f \uparrow_{\oplus_{F} P_{\alpha}}$, so that each $K_{F}$ is projective, since $f\left(\bigoplus_{F} P_{\alpha}\right) \in \mathcal{Y}_{0}$. But then $K=\bigcup_{F \subset I} K_{F}$ is a direct limit of projective modules, and so is projective since $R$ is perfect. Thus proj $\operatorname{dim} \mathcal{Y} \leq 1$.

We also note that a proof similar to the one for Claim 7.6 yields

Claim 7.8. $\operatorname{Ext}_{R}^{1}\left(\mathcal{Y}_{0}, \mathcal{X}\right)=0$.

So our question becomes one of extending this to $\operatorname{Ext}_{R}^{1}(\mathcal{Y}, \mathcal{X})=0$.

As we mentioned in our introductory remarks, Happel, Reiten and Smalø [14 also characterized quasitilted artin algebras as those of global dimension $\leq 2$ whose finitely generated indecomposable right modules each have either injective or projective dimension at most 1 (so, by duality, any right quasitilted artin algebra is also left quasitilted). Thus we are led to question whether a ring of right global dimension $\leq 2$, each of whose right modules is a direct sum of a module of injective dimension $\leq 1$ and a module of projective dimension $\leq 1$, is a quasitilted ring.

\section{Appendix: Ext And DiRECT Sums}

We do not know if the analogue of the natural isomorphism $\operatorname{Ext}_{R}^{1}\left(\bigoplus_{I} M_{\alpha}, L\right) \cong$ $\Pi_{I} \operatorname{Ext}_{R}^{1}\left(M_{\alpha}, L\right)$ for $R$-modules is valid for infinite sets $I$ and cocomplete abelian categories. However, for the purpose of this paper it will suffice to show that there is an embedding $\operatorname{Ext}_{\mathcal{A}}^{1}\left(\bigoplus_{I} M_{\alpha}, L\right) \longmapsto \Pi_{I} \operatorname{Ext}_{\mathcal{A}}^{1}\left(M_{\alpha}, L\right)$. To this end, assume $\mathcal{A}$ is a cocomplete abelian category and consider an exact sequence

$$
E: \quad 0 \rightarrow L \stackrel{f}{\longrightarrow} N \stackrel{g}{\longrightarrow} \bigoplus_{I} M_{\alpha} \rightarrow 0
$$


with injections $\iota_{a}: M_{a} \rightarrow \bigoplus_{I} M_{\alpha}$, representing an element of $\operatorname{Ext}_{\mathcal{A}}^{1}\left(\bigoplus_{I} M_{\alpha}, L\right)$, also consider the pullback diagrams

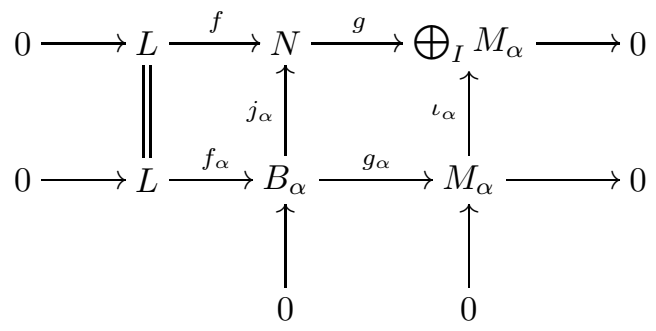

to obtain representatives

$$
E_{\alpha}: \quad 0 \rightarrow L \stackrel{f_{\alpha}}{\longrightarrow} B_{\alpha} \stackrel{g_{\alpha}}{\longrightarrow} M_{\alpha} \rightarrow 0
$$

of $\operatorname{Ext}_{\mathcal{A}}^{1}\left(M_{\alpha}, L\right)$, and let $\Theta(E)=\left(E_{\alpha}\right)_{I} \in \Pi_{I} \operatorname{Ext}_{\mathcal{A}}^{1}\left(M_{\alpha}, L\right)$.

To see that $\Theta$ is additive, consider the commutative diagram

$$
\begin{aligned}
& 0 \rightarrow L \oplus L \stackrel{f_{\alpha} \oplus f_{\alpha}^{\prime}}{\longrightarrow} \quad B_{\alpha} \oplus B_{\alpha}^{\prime} \quad \stackrel{g_{\alpha} \oplus g_{\alpha}^{\prime}}{\longrightarrow} \quad M_{\alpha} \oplus M_{\alpha} \quad \rightarrow 0 \\
& \| \quad j_{\alpha} \oplus j_{\alpha}^{\prime} \downarrow \quad \iota_{\alpha} \oplus \iota_{\alpha}^{\prime} \downarrow \\
& 0 \rightarrow L \oplus L \quad \stackrel{f \oplus f^{\prime}}{\longrightarrow} \quad N \oplus N^{\prime} \quad \stackrel{g \oplus g^{\prime}}{\longrightarrow}\left(\bigoplus_{I} M_{\alpha}\right) \oplus\left(\bigoplus_{I} M_{\alpha}\right) \rightarrow 0
\end{aligned}
$$

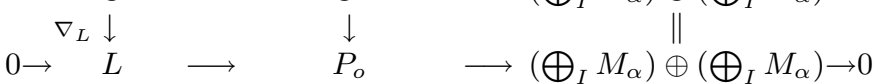

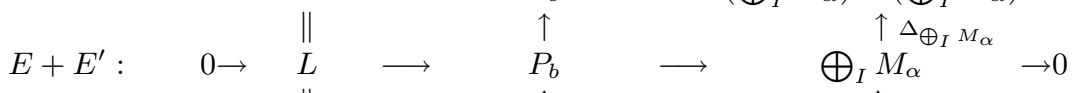

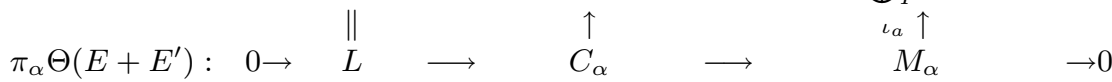

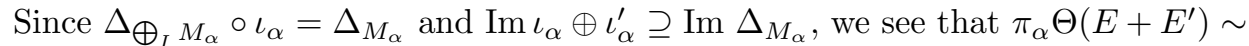
$\pi_{\alpha} \Theta(E)+\pi_{\alpha} \Theta\left(E^{\prime}\right)$, and so $\Theta$ is indeed additive.

To show that $\Theta$ is well defined, suppose that $E$ splits with $g i=1_{\oplus_{I}} M_{\alpha}$. Then

$$
i \iota_{\alpha}: M_{\alpha} \rightarrow N \quad \text { and } \quad 1_{M_{\alpha}}: M_{\alpha} \rightarrow M_{\alpha}
$$

with

$$
g i \iota_{\alpha}=\iota_{\alpha} 1_{M_{\alpha}} .
$$

Thus there is a unique morphism $k_{\alpha}: M_{\alpha} \rightarrow B_{\alpha}$ with

$$
\iota_{\alpha} k_{\alpha}=1_{M_{\alpha}}
$$

(and $j_{\alpha} k_{\alpha}=i \iota_{\alpha}$ ) and so every $E_{\alpha}$ splits. Thus $\Theta$ is well defined.

Now suppose that each

$$
E_{\alpha}: \quad 0 \rightarrow L \stackrel{f_{\alpha}}{\longrightarrow} B_{\alpha} \stackrel{g_{\alpha}}{\longrightarrow} M_{\alpha} \rightarrow 0
$$

splits with some $k_{\alpha}: M_{\alpha} \rightarrow B_{\alpha}$ such that

$$
g_{\alpha} k_{\alpha}=1_{M_{\alpha}} .
$$

Then there is a unique morphism

$$
i: \bigoplus_{I} M_{\alpha} \rightarrow N
$$

with

$$
i \iota_{\alpha}=j_{\alpha} k_{\alpha}
$$


and hence

$$
g i i_{\alpha}=g j_{\alpha} k_{\alpha}=\iota_{\alpha} g_{\alpha} k_{\alpha}=\iota_{a} .
$$

Thus $g i=1_{\oplus_{I} M_{\alpha}}$, and so $E$ splits.

Now we have proved

Proposition 8.1. $\Theta: \operatorname{Ext}_{\mathcal{A}}^{1}\left(\bigoplus_{I} M_{\alpha}, L\right) \rightarrow \Pi_{I} \operatorname{Ext}_{\mathcal{A}}^{1}\left(M_{\alpha}, L\right)$ is a monomorphism of abelian groups.

If $I$ is finite then $\Theta$ is an isomorphism.

Proposition 8.2. If $F$ is a finite set and $\mathcal{A}$ is an arbitrary abelian category, then $\Theta: \operatorname{Ext}_{\mathcal{A}}^{1}\left(\bigoplus_{F} M_{\alpha}, L\right) \rightarrow \Pi_{F} \operatorname{Ext}_{\mathcal{A}}^{1}\left(M_{\alpha}, L\right)$ is a isomorphism of abelian groups.

Proof. Given exact sequences

$$
E_{\alpha}: \quad 0 \rightarrow L \stackrel{f_{\alpha}}{\longrightarrow} B_{\alpha} \stackrel{g_{\alpha}}{\longrightarrow} M_{\alpha} \rightarrow 0
$$

for $\alpha=1,2$, consider the pushout diagram

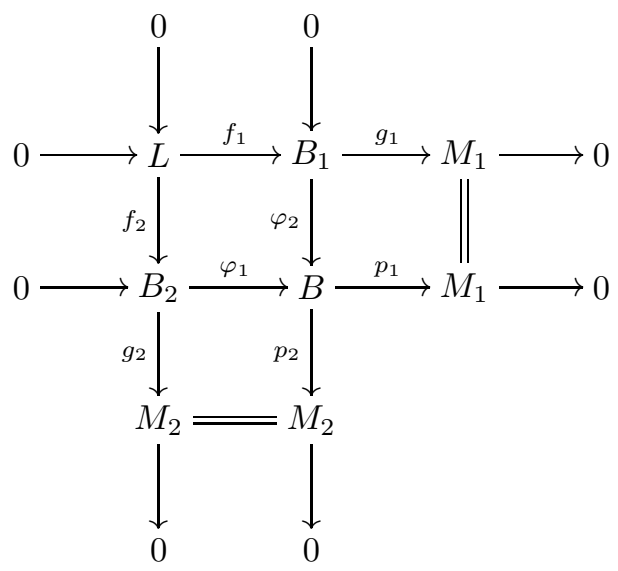

and let $p$ be the product morphism

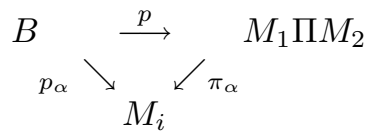

Then $\pi_{1} p \varphi_{2} f_{1}=p_{1} \varphi_{1} f_{2}=0$, and similarly $\pi_{2} p \varphi_{1} f_{2}=0$. Thus $\operatorname{Im} \varphi_{2} f_{1} \subseteq \operatorname{Ker} p$. On the other hand, if $K \stackrel{\varphi}{\longrightarrow} B$ is the kernel of $p$, then, since $B_{1} \stackrel{\varphi_{2}}{\longrightarrow} B$ is the kernel of $p_{2}$ and $p_{2} \varphi=0$ there is a commutative diagram

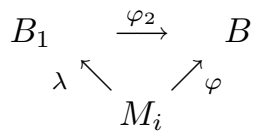

(with unique $\lambda$ ). Now

$$
0=p_{1} \varphi=p_{1} \varphi_{2} \lambda=g_{1} \lambda
$$

so, since $L \stackrel{f_{1}}{\longrightarrow} B_{1}$ is the kernel of $g_{1}$, there is a unique $\lambda^{\prime}: K \rightarrow L$ with $f_{1} \lambda^{\prime}=\lambda$. Thus

and

$$
\varphi_{2} f_{1} \lambda^{\prime}=\varphi_{2} \lambda=\varphi
$$

$$
\operatorname{Im} \varphi_{2} f_{1} \supseteq \operatorname{Im} \varphi_{2} f_{1} \lambda^{\prime}=\operatorname{Im} \varphi=\operatorname{Ker} p
$$


So we have an exact sequence

$$
E: \quad 0 \rightarrow L \stackrel{\varphi_{2} f_{1}}{\longrightarrow} B \stackrel{p}{\longrightarrow} M_{1} \oplus M_{2} \rightarrow 0
$$

with $E \in \operatorname{Ext}_{\mathcal{A}}^{1}\left(M_{1} \oplus M_{2}\right)$. Finally, upon checking that the diagram

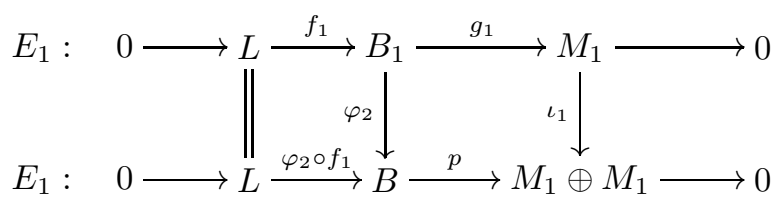

commutes, we see that $\pi_{1} \Theta E \sim E_{1}$, and similarly $\pi_{2} \Theta E \sim E_{2}$.

Corollary 8.3. Let $\mathcal{A}$ be a cocomplete abelian category. If $\operatorname{Ext}_{\mathcal{A}}^{1}(V, L)=0$ and $P \in \operatorname{Add}(V)$, then $\operatorname{Ext}_{\mathcal{A}}^{1}(P, L)=0$.

\section{ADDED IN PROOF}

(1) The article with E. Gregorio mentioned in the paragraph following the proof of Proposition 7.5 has appeared in Colloq. Math., 104, 151-156, 2006, MR2195804.

(2) In Symposia Mathematica, vol. XXIII, 321-412, Instituto Naz. Alta Mat., 1979, MR0565613 (81i:16032), C. M. Ringel proved that if $R$ is a finite-dimensional hereditary algebra, the preinjective modules form a torsion class $\mathcal{X}_{0}$ in mod- $R$ that generates a torsion theory $(\mathcal{X}, \mathcal{Y})$ in Mod- $R$ that splits if and only if $R$ is tame. This fact provides a negative answer to our question preceding Claim 7.6 .

\section{REFERENCES}

[1] F. W. Anderson and K. R. Fuller. Rings and Categories of Modules. Springer-Verlag, Inc., New York, Heidelberg, Berlin, second edition, 1992. MR.1245487 (94i:16001)

[2] K. Bongartz. Tilted algebras. "Proc. ICRA III (Puebla, 1980)", LNM 903, Springer, 26-38, 1981. MR0654701 (83g:16053)

[3] S. Brenner and M. Butler. Generalizations of the Bernstein-Gelfand-Ponomarev reflection functors. "Proc. ICRA II (Ottawa, 1979)", LNM 832, Springer, 103-169, 1980. MR0607151 (83e:16031)

[4] R. R. Colby and K. R. Fuller. Tilting, cotilting and serially tilted rings. Comm. Algebra, 18, 1585-1615, 1990. MR:1059750 (91h:16011)

[5] R. R. Colby and K. R. Fuller. Tilting and torsion theory counter equivalences. Comm. Algebra, 23, 4833-4849, 1995. MR1356105 (96k:16015)

[6] R. R. Colby and K. R. Fuller. Equivalence and Duality for Module Categories. Cambridge University Press, 2004. MR2048277 (2005d:16001)

[7] R. Colpi. Tilting in Grothendieck Categories. Forum Math., 11, 735-759, 1999. MR.1725595 (2000h:18018)

[8] R. Colpi and J. Trlifaj. Tilting modules and tilting torsion theories. J. Algebra, 178, 614-634, 1995. MR1359905 (97e:16003)

[9] S. E. Dickson. A torsion theory for abelian categories. Trans. Amer. Math. Soc., 121, 223-235, 1966. MR0191935 (33:162)

[10] E. E. Enochs and O. M. Jenda. Relative homological algebra. Walter de Gruyter \& Co., Berlin, 2000. MR:1753146 (2001h:16013)

[11] C. Faith. Rings with ascending condition on annihilators. Nagoya Math. J., 27, 179-191, 1966. MR0193107 (33:1328)

[12] R. Fossum, P. Griffith, I. Reiten. Trivial Extensions of Abelian Categories. Springer-Verlag Lect. Notes in Math. 456, 1975. MR0389981 (52:10810)

[13] D. Happel and I. Reiten. An introduction to quasitilted algebras. An. St. Univ. Ovidius Constanta, 4, 137-149, 1996. MR 1428462 (98g:16009)

[14] D. Happel, I. Reiten, S. O. Smalø. Tilting in Abelian Categories and Quasitilted Algebras. Memoirs of the A.M.S., vol. 575, 1996. MR.1327209 (97j:16009) 
[15] D. Happel and C. M. Ringel. Tilted algebras. Trans. Amer. Math. Soc., 274, 399-443, 1982. MR0675063 (84d:16027)

[16] B. Keller. Derived Categories and Tilting (to appear in Handbook of Tilting Theory).

[17] C. Menini and A. Orsatti. Representable equivalences between categories of modules and applications. Rend. Sem. Mat. Univ. Padova, 82, 203-231, 1989. MR.1049594 (91h:16026)

[18] B. Mitchell. Theory of Categories. Academic Press, London and New York, 1965. MR0202787 $(34: 2647)$

[19] Y. Miyashita. Tilting modules of finite projective dimension. Math. Z., 193, 113-146, 1986. MR0852914 (87m:16055)

[20] N. Popescu. Abelian Categories with applications to Rings and Modules. Academic Press, London and New York, 1973. MR0340375 (49:5130)

[21] L. Small. An example in noetherian rings. Proc. Natl. Acad. Sci. USA, 54, 1035-1036, 1965. MR0188252 (32:5691)

[22] Bo Stentröm. Rings of Quotients. Springer-Verlag, Berlin, Heidelberg, New York, 1975. MR0389953 (52:10782)

Department of Pure and Applied Mathematics, University of Padova, via Belzoni 7 , I 35100 PADOVA, ItALY

E-mail address: colpi@math.unipd.it

Department of Mathematics, University of Iowa, Iowa City, Iowa 52242-1419

E-mail address: kfuller@math.uiowa.edu 